TRANSACTIONS OF THE

AMERICAN MATHEMATICAL SOCIETY

Volume 350, Number 8, August 1998, Pages 3021-3041

S 0002-9947(98)02226-0

\title{
SMALL SUBALGEBRAS OF STEENROD AND MORAVA STABILIZER ALGEBRAS
}

\author{
N. YAGITA
}

\begin{abstract}
Let $P(j)$ (resp. $\left.S(n)_{(j)}\right)$ be the subalgebra of the Steenrod algebra $\mathcal{A}_{p}$ (resp. $n$th Morava stabilizer algebra) generated by reduced powers $\mathcal{P}^{p^{i}}, 0 \leq i \leq j$ (resp. $t_{i}, 1 \leq i \leq j$ ). In this paper we identify the dual $P(j-1)^{*}$ of $P(j-1)$ (resp. $S(n)_{(j)}$, for $j \leq n$ ) with some Frobenius kernel (resp. $F_{p^{n}}$-points) of a unipotent subgroup $G(j+1)$ of the general linear algebraic group $G L_{j+1}$. Using these facts, we get the additive structure of $H^{*}(P(1))=\operatorname{Ext}_{P(1)}(Z / p, Z / p)$ for odd primes.
\end{abstract}

\section{INTRODUCTION}

The Adams and Adams-Novikov spectral sequences are the most powerful tools to compute stable homotopy groups of spheres. The $E_{2}$-terms of these spectral sequences are the cohomology of the Steenrod algebra or reduce essentially to the cohomology of the Morava stabilizer algebra. We study here the cohomology of some small subalgebras of these algebras for odd primes $p$. Let $P(j)$ be the subalgebra of the $\bmod p$ Steenrod algebra $\mathcal{A}_{p}$, generated by the reduced powers $\mathcal{P}^{p^{i}}, 0 \leq i \leq j$, and let $S(n)_{(j)}$ be the subalgebra of the $n$th Morava stabilizer algebra, generated by $t_{i}, 0<i \leq j$. We will examine a unipotent subgroup $G(j+1)$ of the general linear algebraic group $\mathrm{GL}_{j+1}$, generated by matrices $\left\{\left(a_{i j}=a_{i-j, 1}^{p^{j-1}}\right)\right\}$ with $a_{i i}=1$, $a_{i j}=0$ for $i<j$. Let $k[G(j+1)]$ be its coordinate ring with coefficients the algebraic closure $k=\bar{F}_{p}$. Then the dual $P(j)^{*} \otimes k$ of $P(j) \otimes k$ is a quotient of $k[G(j+2)]$ by images of Frobenius maps (a modified Frobenius kernel), and if $j \leq n$, then $S(n)_{(j)} \otimes k$ is the dual of the group ring $k\left(G(j+1)\left(F_{p^{n}}\right)\right)$ of $F_{p^{n}}$-points, and is the quotient of $k[G(j+1)]$ by the ideal $\left(x^{p^{n}}-x\right)$.

Hereafter we will always work in $P(j) \otimes k$, so we will leave out the $k$ (and the same for $S(n))$. The cohomology $H^{*}(-)$ will always mean $H^{*}(-; k)$.

In $\S 1$ of this paper, we state the relations between this algebraic group and the Steenrod and Morava stabilizer algebras. In $\S 2$ and $\S 3$, we consider the cohomology of the Frobenius kernel and of the $F_{q}$-points respectively. The cohomology $H^{*}(P(1))$ is computed for $p=$ odd in $\S 4$ (when $p=2$, it is the classical result of Adams and Liulevicius [Li]), and $H^{*}(P(2))$ is computed by Shimada-Iwai [S-I]). The cohomology $H^{*}\left(S(2)_{(2)}\right)$ is computed in $\S 5$. The last section is a description of the cohomology of $S(2)$, which was already studied by Ravenel [R 1]-[R 3], Henn $[\mathrm{H}]$ and Gorbounov-Siegel-Symonds [G-S-S].

Received by the editors January 9, 1995 .

1991 Mathematics Subject Classification. Primary 55N22; Secondary 57R77.

(C)1998 American Mathematical Society 
We do not directly use theories of algebraic groups and their representations in these concrete cases computations. However these computations are suggested by those for the maximal unipotent group $U$ in $\mathrm{GL}_{3}$, which was done in joint work with Kaneda, Shimada and Tezuka [K-S-T-Y 1]. K. Shimomura and C. Peterson suggested the importance of algebraic groups for the Morava stabilizer algebra. H. Miller pointed out the similarity of cohomology of $P(1)$ and the extraspecial p-group $U\left(F_{p}\right)$, which was the start of this work. The author thanks those people.

\section{Algebraic groups and the Steenrod Algebra}

Let $k$ be the algebraic closure of $F_{p}$ for an odd prime. We think of an algebraic group $G$ as a functor $G: A \rightarrow G(A)$ from commutative $k$-algebras $A$ to groups $G(A)$. For example, the additive group $G_{a}$ is defined by $G_{a}(A)=A$. In this paper, we consider the unipotent algebraic subgroup $G(n+1)$ of the general linear group $\mathrm{GL}_{n+1}$, generated by matrices (see [K-S-T-Y 2], 3.3)

$$
g\left(a_{1}, \ldots, a_{n}\right)=\left(\begin{array}{cccccc}
1 & & & & \\
a_{1} & 1 & & & \\
a_{2} & a_{1}^{p} & 1 & & 0 \\
a_{3} & a_{2}^{p} & a_{1}^{p^{2}} & 1 & \\
& & & & \\
a_{n} & a_{n-1}^{p} & & & a_{1}^{p^{n-1}}
\end{array}\right) .
$$

The coordinate ring $k[G]$ is a Hopf algebra defined by the homomorphisms of setvalued functors $\operatorname{Hom}\left(G, G_{a}\right)$. Taking $\xi_{i}: g\left(a_{1}, \ldots, a_{n}\right) \rightarrow a_{i}$, its coordinate ring is $k[G(n+1)] \cong k\left[\xi_{1}, \ldots, \xi_{n}\right]$ with the diagonal $\psi\left(\xi_{i}\right)=\sum_{j=0}^{i} \xi_{j} \otimes \xi_{i-j}^{p^{j}}, \xi_{0}=1$. For each element in the coordinate ring, we assign a weight by $\operatorname{wt}\left(\xi_{i}\right)=p^{i}-1$, indeed, the conjugation action of the diagonal matrix $t=\operatorname{diag}\left(b, b^{p}, \ldots, b^{p^{n-1}}\right)$ is represented as $\operatorname{ad}(t) g\left(a_{1}, \ldots, a_{n}\right)=g\left(b^{p-1} a_{1}, \ldots, b^{p^{n}-1} a_{n}\right)$.

On the other hand, it is well known that the dual $P(\infty)^{*}$ of the subalgebra of Steenrod algebra $\mathcal{A}_{p} \otimes k$, generated by reduced powers, is isomorphic to $k\left[\xi_{1}, \ldots, \xi_{n}, \ldots\right] \cong k[G(\infty)]$ with the same diagonal map. Moreover the degree $\operatorname{deg}\left(\xi_{i}\right)=2\left(p^{i}-1\right)=2 \mathrm{wt}\left(\xi_{i}\right)$, twice the weight. Hence we may think that for a topological space $X$, the ordinary homology $H_{*}(X)$ is a $k\left[G(\infty) \ltimes G_{m}\right]$-comodule where $G_{m}$ is the multiplicative group, and $i$-dimensional cohomology $H^{i}(X)$ is the weight $i$-space, i.e. $b(x)=b^{i} x$ for $x \in H^{i}(X)$ and $b \in G_{m}(k)=k^{*}$.

Let $P(n-1)$ be the sub-Hopf algebra of $P(\infty)$ generated by reduced powers $\mathcal{P}^{p^{i}}$, $0 \leq i \leq n-1$. Then its dual is

$$
P(n-1)^{*} \cong k\left[\xi_{1}, \ldots, \xi_{n}\right] /\left(\xi_{1}^{p^{n}}, \xi_{2}^{p^{n-1}}, \ldots, \xi_{n}^{p}\right) .
$$

For an algebraic group $G$, the Frobenius kernel $G_{r}$ is defined as the functor which represents the kernel of the $r$ th Frobenius map $\sigma^{r}: G \rightarrow G$. Its coordinate ring $k\left[G_{r}\right]$ is represented as $k[G] /\left(x^{p^{r}} \mid x \in\right.$ augmentation ideal $)$. Therefore $P(n-1)^{*}$ is a quotient algebra of $k\left[G(n+1)_{n}\right]$, indeed we have

Theorem 1.3. Let $\mathrm{GP}(n+1)$ be the functor taking

$$
A \rightarrow g\left(G_{a}(A)_{n}, \ldots, G_{a}(A)_{1}\right), \quad G_{a}(A)_{i}=\left(\operatorname{Ker} \sigma^{i} \mid A\right) .
$$

Then $k[\mathrm{GP}(n+1)] \cong P(n-1)^{*}$. 
Remark. The subalgebra $P(n)$ of $\mathcal{A}_{p}$ itself is identified with the distribution $\operatorname{Dist}(G(n+2))$ (see 3.3 in [K-S-T-Y 2]).

Next consider the relation between $G(n+1)$ and the Morava stabilizer algebra. Let $K(n)_{*}(-)$ be Morava $K$-theory with coefficients $K(n)_{*}=Z / p\left[v_{n}, v_{n}^{-1}\right]$ with $\left|v_{n}\right|=2\left(p^{n}-1\right)$. Recall

$$
\begin{aligned}
& K(n)_{*} K(n) \cong K(n)_{*}\left[t_{1}, \ldots t_{i}, \ldots\right] /\left(v_{n} t_{i}^{p^{n}}-v_{n}^{p^{n}} t_{i}\right) \quad \text { and } \\
& S(n)=K(n)_{*} K(n) /\left(v_{n}-1\right) \otimes k=k\left[t_{1}, \ldots\right] /\left(t_{i}^{p^{n}}-t_{i}\right) .
\end{aligned}
$$

The diagonal map in $S(n)$ is given ([R 1], (3.1))

$$
\begin{gathered}
\psi\left(t_{i}\right)=\sum_{0 \leq j \leq i} t_{j} \otimes t_{i-j}^{p^{j}} \quad \text { for } i \leq n, \\
\psi\left(i_{n+i}\right)=\sum_{0 \leq j \leq n+i} t_{j} \otimes t_{i+n-j}^{p^{j}}-C_{p^{n}}\left(t_{i} \otimes 1, t_{i-1} \otimes t_{1}^{p}, \ldots, 1 \otimes t_{i}\right)
\end{gathered}
$$

where $C_{p^{n}}\left(x_{1}, \ldots x_{k}\right)$ is the $\bmod p$ reduction of $p^{-1}\left(\left(\sum x_{i}\right)^{p^{n}}-\sum\left(x_{i}^{p^{n}}\right)\right)$. Let us write the sub-Hopf algebra

$$
S(n)_{(j)}=k\left[t_{1}, \ldots, t_{j}\right] /\left(t_{i}^{p^{n}}-t_{i} \mid 1 \leq i \leq j\right) \subset S(n) .
$$

One of the important theorems of Morava ([R 1], [R 2]) is the following. There exists a profinite group $S_{n}$ such such that its group ring $k\left(S_{n}\right)$ is isomorphic to $S(n)^{*}$ where $S(n)^{*}$ is the topological dual of $S(n)$ filtered by $S(n)_{(j)}$.

On the other hand, Kaneda-Radford-Tezuka found (Theorem 2.2.2 in [T]) the relation between the group ring of the group of $F_{q}$-points and the coordinate ring; if $G$ is a unipotent algebraic group defined over $F_{p}$, then the dual of the group ring of the finite group of its $F_{q}$-points is a quotient ring of the coordinate ring

$$
k\left(G\left(F_{p^{n}}\right)\right)^{*} \cong k[G] /\left(x^{p^{n}}-x \mid x \in \text { augmentation ideal }\right) .
$$

Hence from (1.5), (1.6) and (1.7), we get

Theorem 1.8. If $j \leq n$, then $k\left(G(j+1)\left(F_{p^{n}}\right)\right)^{*} \cong S(n)_{(j)}$. Therefore we have $H^{*}\left(S(n)_{(j)}\right) \cong H^{*}\left(G(j+1)\left(F_{p^{n}}\right)\right)$.

The weight for elements in $k\left(G(j+1)\left(F_{p^{n}}\right)\right)^{*}$ is given by the action

$$
\operatorname{ad}(t) g\left(a_{1}, \ldots, a_{n}\right)=g\left(b^{p-1} a_{1}, \ldots, b^{p^{n}-1} a_{n}\right)
$$

for $t=\operatorname{dig}\left(b, \ldots, b^{p^{n}-1}\right)$. Here $b \in k^{*}$ such that $b^{p-1} \in F_{p^{n}}^{*}$. So the weight takes the value with modulo $\left(p^{n}-1\right)(p-1)$. However if $n<j$, then the weight takes only value with modulo $\left(p^{n}-1\right)$ by the restrictions for the coproduct $\psi\left(t_{n+i}\right)$. Hence the degree of $K(n)^{*}(X)$ is $2\left(p^{n}-1\right)$-periodic, and the action of $b \in F_{p^{n}}^{*}$ corresponds to the Adams operation.

If $j>n$, then the cohomology of $G(j+1)\left(F_{p^{n}}\right)$ and $S(n)_{(j)}$ are quite different. Consider the case $n=1$ and $j=\infty$. We have the spectral sequence

$$
E_{1} \cong \bigotimes_{i=1}^{\infty} k\left[b_{i}\right] \otimes \bigwedge\left(h_{i}\right) \Rightarrow H^{*}(S(1))
$$

where $h_{i}=\left[t_{i}\right]$ and $b_{i}$ is its Bockstein. The coproduct (1.5) shows $d_{1} h_{i+1}=b_{i}$. So we know

$$
H^{*}(S(1)) \cong \bigwedge\left(h_{1}\right), \quad[\mathrm{R} 1]
$$


On the other hand $G(j+1)\left(F_{p}\right)=V(j+1)\left(F_{p}\right)$ where $V(j+1) \subset \mathrm{GL}_{j+1}$ is the commutative unipotent group generated by matrices $\left(a_{i j}=a_{i-j, 1}\right)$ with $a_{i i}=$ $1, a_{i j}=0$ for $i<j$. We already know [P-Y] the decomposition $V(j+1)=\prod W_{m}$ (see (9) in $[\mathrm{P}-\mathrm{Y}]$ ) to Witt vectors, and the cohomology of Witt vectors $W_{m}$. Thus we get

$$
H^{*}\left(G(\infty)\left(F_{p}\right)\right)=\operatorname{Lim}_{j \rightarrow \infty} H^{*}\left(G(j)\left(F_{p}\right)\right) \cong \bigwedge\left(h_{i} \mid p \nmid i\right) .
$$

\section{Cohomology of the Frobenius Kernel}

First, we study the cohomology of $G(3)$. There is a central extension

$$
1 \rightarrow G_{a} \rightarrow G(3) \rightarrow G(2) \rightarrow 1
$$

where $G_{a}$ (resp. $\left.G(2)\right)$ is generated by $g\left(0, a_{2}\right)$ (resp. $g\left(a_{1}, 0\right)$ ). This induces the spectral sequence

$$
E_{2}^{*, *}=H^{*}(G(2)) \otimes H^{*}\left(G_{a}\right) \Rightarrow H^{*}(G(3)) .
$$

Here the $E_{2}$-term is isomorphic to

$$
E_{2}^{*, *}=\bigotimes_{i=0}^{\infty} k\left[b_{1 i}, b_{2 i}\right] \otimes \wedge\left(h_{1 i}, h_{2 i}\right)
$$

where we identify $h_{j i}=\left[\xi_{j}^{p^{i}}\right]$ and $b_{j i}=\tilde{\mathcal{B}} h_{j i}=\left[p^{-1} \sum_{1 \leq k \leq p-1}\left(\begin{array}{c}p \\ k\end{array}\right) \xi_{j}^{p^{i}(p-k)} \otimes \xi_{j}^{p^{i} k}\right]$ in the cobar complex. Here $\widetilde{\mathcal{B}}=\mathcal{B} \sigma$ is the Bockstein defined in the cobar complex (see Appendix 1 in [R 3]), which is the composition of the Frobenius map $\sigma$ and the usual Bockstein $\mathcal{B}$.

Remark. In the notation [Y], [K-S-T-Y 1] $h_{i j}=x_{i}(j)$ and $b_{i j}=y_{i}(j+1)$.

Since $\psi\left(\xi_{2}\right)-\left(\xi_{2} \otimes 1+1 \otimes \xi_{2}\right)=\xi_{1} \otimes \xi_{1}^{p}$, the first differential of the spectral sequence is

$$
d_{2} h_{20}=h_{10} h_{11} .
$$

From the naturality of $\widetilde{\mathcal{B}}$ and the fact $\widetilde{\mathcal{P}}^{0}=\sigma$, we know

$$
\begin{aligned}
d_{3} b_{20} & =\widetilde{\mathcal{B}} d_{2} h_{20}=\widetilde{\mathcal{B}}\left(h_{10}\right) \widetilde{\mathcal{P}}^{0}\left(h_{11}\right)-\widetilde{\mathcal{P}}^{0}\left(h_{10}\right) \widetilde{\mathcal{B}}\left(h_{11}\right) \\
& =b_{10} h_{12}-b_{11} h_{11} .
\end{aligned}
$$

By using reduced powers $\widetilde{\mathcal{P}}^{i}$ in the cobar complexes (see also Appendix 1 in [R 3]) and the Cartan-Serre transgression theorem, we get

$$
\begin{aligned}
d_{2 p^{n}+1} b_{20}^{p^{n}} & =\widetilde{\mathcal{P}}^{p^{n-1}} \ldots \widetilde{\mathcal{P}} d_{3} b_{20} \\
& =b_{10}^{p^{n}} h_{1, n+2}-b_{11}^{p^{n}} h_{1, n+1} .
\end{aligned}
$$

The Kudo transgression theorem implies

$$
\begin{aligned}
& d_{2 p^{n}(p-1)+1}\left(b_{20}^{p^{n}(p-1)} \otimes d_{2 p^{n}+1} b_{20}^{p^{n}}\right)=\widetilde{\mathcal{B}} \widetilde{\mathcal{P}}^{p^{n}}\left(d_{2 p^{n}+1} b_{20}^{p^{n}}\right), \\
& =b_{10}^{p^{n+1}} b_{1, n+2}-b_{1, n+1} b_{11}^{p^{n+1}} .
\end{aligned}
$$

Therefore by acting with the Frobenius map, we know that

$$
J=\left(b_{1 i}^{p^{m+1}} b_{1, m+i+2}-b_{1, i+1}^{p^{m+1}} b_{1, m+i+1}\right)
$$

is zero in $H^{*}(G(3))$. 
Next consider the cohomology of $G(3)_{r}$. In this case $b_{k i}=h_{k i}=0$ if $i \geq r$. Hence the $E_{2}$-term of the spectral sequence type of (2.1) but converging to $H^{*}\left(G(3)_{r}\right)$ is

$$
E_{2}^{*, *} \cong \bigotimes_{0 \leq i \leq r-1} k\left[b_{1 i}, b_{2 i}\right] \otimes \bigwedge\left(h_{1 i}, h_{2 i}\right)
$$

From (2.5) we know that $b_{20}^{p^{r-1}}$ is a permanent cycle. By using the Frobenius maps, we also see $b_{2 j}^{p^{r-j-1}}$ is a permanent cycle.

Theorem 2.9. Let $\tilde{b}_{2 j} \in H^{*}\left(G(3)_{r}\right)$ be an element which corresponds to $b_{2 j}^{p^{r-j-1}}$ in the spectral sequence (2.8). Then $H^{*}\left(G(3)_{r}\right)$ is finitely generated over $k\left[\tilde{b}_{2 j} \mid 0 \leq\right.$ $j \leq r-1] \otimes k\left[b_{10}\right]$. Moreover $b_{1 j}^{(p+1)^{r-j}}=0$ for $j \geq 1$.

Proof. From (2.7), $b_{1, k-1}^{p} b_{1, k-1}-b_{1, k-2}^{p} b_{1, k}=0$. Take $k=r$, and we get $b_{1, r-1}^{p+1}=0$. Since $b_{1, k-2}^{p+1}=\left(b_{1, k-3}\right)^{p} b_{1, k-1}$, we also have $\left(b_{1, r-2}\right)^{(p+1)^{2}}=0$. By induction on $r$, we can easily see the last statement.

Let $B_{1}=\bigotimes_{0 \leq i \leq r-1} k\left[b_{1 i}\right]$ and $B_{2}=\bigotimes_{0 \leq i \leq r-1} k\left[b_{2 i}^{p^{r-j-1}}\right]$. Then we can write

$$
E_{2}^{*, *} \cong A \otimes B_{2} \text { where } A \text { is a finitely generated } B_{1} \text {-module. }
$$

Since $d \mid B_{2}=0$, the cohomology $E_{3}^{*, *}=H^{*}\left(A \otimes B_{2}, d_{2}\right)=H\left(A, d_{2}\right) \otimes B_{2}$. It is a subquotient ring of the Noetherian ring $E_{2}^{*, *}$, hence it is also Noetherian. Therefore $E_{s}^{*, *}$ is Noetherian for finite $s$. Since $A^{*, t}=0$ for $t>\sum\left|b_{2 i}^{p^{r-j-1}}\right|$, we get $E_{t} \cong E_{\infty}$. This shows that $H^{*}\left(G(3)_{r}\right)$ is finite over $B_{1} \otimes B_{2}$. q.e.d.

Next consider $G(n+1)_{r}$. The filtration given by maps

$$
G(2) \leftarrow G(3) \leftarrow \cdots \leftarrow G(n+1) \leftarrow G_{a}
$$

induces a spectral sequence

$$
E_{1}^{*}=\bigotimes_{1 \leq i \leq n} \bigotimes_{0 \leq j \leq r-1} k\left[b_{i j}\right] \otimes \bigwedge\left(h_{i j}\right) \Rightarrow H\left(G(n+1)_{r}\right) .
$$

Before considering this spectral sequence, we study more general cases. Let $U$ be a unipotent algebraic group and

$$
1 \rightarrow G_{a} \rightarrow U \rightarrow U^{\prime} \rightarrow 1
$$

be a central extension of $U^{\prime}$. The induced spectral sequence for $H^{*}\left(U_{r}\right)$ has the $E_{2}$-term

$$
E_{2}^{*, *}=H^{*}\left(U_{r}^{\prime}\right) \otimes\left(\bigotimes_{0 \leq j \leq r-1} k\left[b_{j}\right] \otimes \bigwedge\left(h_{j}\right)\right) \Rightarrow H^{*}\left(U_{r}\right) .
$$

Lemma 2.13. There is $s>0$ such that $b_{i}^{p^{s}}$ is a permanent cycle in the spectral sequence above. Hence $H^{*}\left(U_{r}\right)$ is Noetherian.

Proof. By induction we assume $H^{*}\left(U_{r}^{\prime}\right)$ is Noetherian. The transgression theorem shows $d_{2 p^{i}+1} b_{t^{p^{i}}}=a_{i, t} \in H\left(U_{r}^{\prime}\right)$. Since $H\left(U_{r}^{\prime}\right)$ is Noetherian, there is $s_{t}$ such that $a_{k t} \in\left(a_{0 t}, \ldots, a_{s t}\right)$ for all $k>s_{t}$. This means $b_{t}^{p^{k}}$ is a permanent cycle. Then by arguments similar to the last parts of the proof of Theorem 2.9, we can prove the lemma. q.e.d. 
Theorem 2.14. In the spectral sequence (2.11), there is an $s>0$ such that $b_{i j}^{p^{s}}+z$ is a permanent cycle where $z \in k\left[b_{i k} \mid k>j\right]$. Let us write $\tilde{b}_{i j}$ for a corresponding element in $H^{*}\left(G(n+1)_{r}\right)$. Then $H^{*}\left(G(n+1)_{r}\right)$ is finite over $k\left[\tilde{b}_{i j} \mid i>j\right.$ or $i>$ $[n / 2]]$.

Proof. The first statement is immediate from Lemma 2.13. Let us write $G(n, i)$ for the kernel of the natural projection $G(n) \rightarrow G(i)$. Then we have the spectral sequences

$$
\begin{gathered}
E_{2}^{*, *} \cong H^{*}\left(G(n)_{r}\right) \otimes H^{*}\left(G_{a r}\right) \Rightarrow H^{*}\left(G(n+1)_{r}\right), \\
E(i)_{2}^{*, *} \cong H^{*}\left(G(n, i)_{r}\right) \otimes H^{*}\left(G_{a r}\right) \Rightarrow H^{*}\left(G(n+1, i)_{r}\right)
\end{gathered}
$$

and induced map $i^{*}: E_{r}^{*, *} \rightarrow E(i)_{r}^{*, *}$. Take $n=2 i$. Then $\psi\left(\xi_{2 i}\right)-\left(\xi_{2 i} \otimes 1-1 \otimes \xi_{2 i}\right)=$ $\xi_{i} \otimes \xi_{i}^{p^{i}} \bmod \left(\xi_{1}, \ldots, \xi_{i-1}\right)$ implies $d_{2} h_{2 i, 0}=h_{i, 0} h_{i, i}$ in $E(i)_{2}$. Hence by Kudo's transgression theorem

$$
\begin{aligned}
& d_{2 p^{i-1}(p-1)+1}\left(b_{2 i, 0}^{p^{i-1}(p-1)} \otimes d_{2 p^{i-1}+1} b_{2 i, 0}^{p^{i-1}}\right) \\
& =b_{i, i}^{p^{i}+1}-b_{i, 0}^{p^{i}} b_{i, 2 i} \quad \text { in } E(i)_{2 p^{i-1}(p-1)+1} .
\end{aligned}
$$

We can take as $\tilde{b}_{i i}$, the left-hand side of $(1)$ in $H^{*}\left(G(2 i)_{r}\right)$. Indeed, it is zero in $H^{*}\left(G(2 i+1)_{r}\right)$ and the restriction of the image in $H^{*}\left(G(2 i, i)_{r}\right)$ is the right-hand side of (1). By the Frobenius map, we can take $\tilde{b}_{i j}=0$ for all $j \geq i$. q.e.d.

\section{Cohomology of $F_{q}$-POints}

In this section, we assume $q=p^{r}$, and we study cohomology of $G(n+1)\left(F_{q}\right)$ comparing it to that of $G(n+1)_{r}$. First we study the case $G(3)$. The central extension (2.1) induces a spectral sequence such that

$$
E_{2}^{*, *} \cong(2.8) \Rightarrow H^{*}\left(G(3)\left(F_{q}\right)\right)
$$

Here the $E_{2}$-term is isomorphic to (2.8) as rings but the Frobenius map acts differently

$$
\sigma h_{i, r-1}=h_{i, 0}, \sigma b_{i, r-1}=b_{i, 0} .
$$

Hence we take the index $j \in Z / r$ for $h_{i, j}, b_{i, j}$. The differentials (2.3)-(2.6) also hold. In particular the ideal $J$ is zero in $H^{*}\left(G(3)\left(F_{q}\right)\right)$. Before studying this ideal $J$, we recall Quillen's theorem for cohomology of finite groups.

Given a $k$-algebra $R$, let $R(k)$ be the variety of $R$, that is, the set of ring maps $\operatorname{Hom}(R, k)$ endowed with the Zariski topology. Let $V$ be a vector space over $k$. Given an ideal $I$ in the symmetric algebra $S(V)$, the variety $\operatorname{Var}(I)$ is the zeros in $V$. Taking the coordinate homomorphism, $\operatorname{Var}(I) \cong(S(V) / I)(k)$. Quillen's stratification theorem for cohomology of finite groups is

Theorem 3.2. ([Q]) Let $G$ be a finite group and $H(G)=H^{\text {even }}(G ; k)$. Then the variety $H(G)(k)$ has a stratification

$$
H(G)(k) \cong \bigvee_{A \in I E} V_{A}^{+}
$$

where $I E$ is a set of representatives of conjugacy classes of elementary abelian $p$ subgroups $A$ of $G$, and $V_{A}^{+}=i^{*}(A \otimes k)^{+}$for $i: A \subset G$ and $(A \otimes k)^{+}=A \otimes k-$

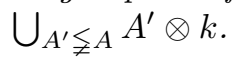


We study maximal elementary abelian $p$-groups in $G(3)\left(F_{q}\right)$. The group $G(3,2)\left(F_{q}\right) \cong\left\{g(0, a) \mid a \in F_{q}\right\} \cong F_{q}$ is the center of $G(3)\left(F_{q}\right)$. The commutator

$$
[g(a, 0), g(b, 0)]=g\left(0, a^{p} b-a b^{p}\right)
$$

(see p. $998[\mathrm{Y}]$, where $\left.g(a, 0)=x_{12}(a) x_{23}\left(a^{p}\right)\right)$. If the subgroup generated by $g(a, 0)$ and $g(b, 0)$ is abelian, then $a^{p} b-a b^{p}=0$, so $a / b=(a / b)^{p}$, hence $a / b \in F_{p}^{*}$. Let $A_{a}$ be the subgroup generated by $g(a, 0)$. Then the conjugacy classes of maximal elementary abelian $p$-groups are written as

$$
A_{a} \oplus G(3,2)\left(F_{q}\right) \cong Z / p \oplus(Z / p)^{r}, \quad \text { with } a \in F_{q}^{*} / F_{p}^{*} .
$$

From Quillen's theorem

Theorem 3.3. $H\left(G(3)\left(F_{q}\right)\right)(k) \cong\left(\bigcup V_{a}\right) \oplus\left(k^{r}\right)$ with $V_{a} \cong A_{a} \otimes k \cong k$ for $a \in$ $F_{q}^{*} / F_{p}^{*}$.

On the other hand, we get

Theorem 3.4. Let $J$ be the ideal (2.7) in $S_{1}=k\left[b_{1 i} \mid 0 \leq i \leq r-1\right]$. Then

$$
\operatorname{Var}(J) \cong \bigcup W_{a} \text { with } W_{a} \cong k, a \in F_{q}^{*} / F_{p}^{*} \text {. }
$$

Proof. Let $\left(b_{0} \cdots b_{r-1}\right) \in \operatorname{Var}(J) \subset(k)^{r}$. Then $b_{0}^{p^{n}} b_{n+1}-b_{1}^{p^{n}} b_{n}=0$ so $\left(b_{1} / b_{0}\right)^{p^{n}}=$ $\left(b_{n+1} / b_{n}\right)$. Let $b_{1} / b_{0}=\lambda$. Then

$$
\left(b_{0}, \ldots, b_{r-1}\right)=\left(b_{0}, \lambda b_{0}, \lambda^{1+p} b_{0}, \ldots, \lambda^{1+p+\cdots+p^{r-2}} b_{0}\right)
$$

and $\lambda^{1+p+\cdots+p^{r-1}}=1$. Since $x^{q-1}-1=0$ for all $x \in F_{q}^{*}$, we can write $\lambda=x^{p-1}$ for $x \in F_{q}^{*}$. Hence the variety is

$$
\operatorname{Var}(J) \cong \bigcup W_{\lambda} \text { for } \lambda \in F_{q}^{*} / F_{p}^{*}
$$

where $W_{\lambda}=\left\{\left(b_{0}, \lambda b_{0}, \ldots, \lambda^{1+p+\cdots+p^{r-2}} b_{0} \mid b_{0} \in k\right\}\right.$. q.e.d.

Lemma 3.5. Let $\pi$ be the projection $\pi: G(3)\left(F_{q}\right) \rightarrow G(2)\left(F_{q}\right)$ and $p: S_{1} / J \rightarrow$ $\pi^{*} H^{*}\left(G(2)\left(F_{q}\right)\right)$ be the induced map. Then $p(k):\left(\pi^{*} H(G(2))\right)(k) \cong \operatorname{Var}(J)$.

Proof. Consider the commutative diagram of $k$-points.



Since $p$ is epic, $p(k)$ is monic. The number of subspaces $V$ and $W$ are the same. Hence $p(k)$ is also epic. q.e.d.

For finite groups $K^{\prime} \subset K$, let $N\left[K^{\prime} \subset K\right]: H^{*}\left(K^{\prime}\right) \rightarrow H^{*}(K)$ be the Evens norm which satisfies naturality and the multiplicative properties (for details see $[E],[Y]$ ). Let us write

$$
\tilde{b}_{2 j}=N\left[G(3,2)\left(F_{q}\right) \subset G(3)\left(F_{q}\right)\right]\left(b_{2 j}\right) .
$$


By the double coset formula, $\tilde{b}_{2 j} \mid G(3,2)\left(F_{q}\right)=b_{2 j}^{p^{r}}$. Then we can define

$$
j: k\left[\tilde{b}_{2 j} \mid 0 \leq j<r\right] \otimes S_{1} /(J) \rightarrow H^{*}\left(G(3)\left(F_{q}\right)\right) .
$$

Theorem 3.7. The above map $j$ is injective modulo $\sqrt{0}$, and finite.

Proof. By Lemma $3.5, j \mid 1 \otimes S_{1} /(J)$ is injective modulo $\sqrt{0}$. By arguments similar to the last part in the proof of Theorem 2.9 , we also see that the spectral sequence for $H *\left(G(3)\left(F_{q}\right)\right)$ also has the form $E_{s}^{*, *} \cong A_{s} \otimes k\left[b_{2 j}^{p^{r}} \mid 0 \leq j<r\right]$ for all $s$ and for some finitely generated $S_{1}$-module $A_{s}$. Thus we have proved the theorem. q.e.d.

Next consider $G(n+1)$. Define the elements

$$
\tilde{b}_{i j}=N\left[G(n+1, i)\left(F_{q}\right) \subset G(n+1)\left(F_{q}\right)\right]\left(b_{i j}\right)
$$

so that $\tilde{b}_{i j} \mid G(n+1, i)\left(F_{q}\right)=b_{i j}^{p^{r i}}$. Then we can easily prove

Theorem 3.8. $H(G(n+1))\left(F_{q}\right)$ is finite over $\otimes k\left[\tilde{b}_{i j}\right]$ where the index $(i j)$ runs over the following (i) and (ii)

(i) $1 \leq i \leq[n / 2]$ and $0 \leq 2 m i \leq j<(2 m+1) i<r$ for some $m$,

(ii) $i>[n / 2]$.

\section{Cohomology of $P(1)^{*}$}

In this section we compute $H^{*}\left(P(1)^{*}\right)$ according to the arguments in $\S 2$. We use $k[G P(3)] \cong P(1)^{*}$ and consider the spectral sequence type (2.8)

$$
E_{2}^{*, *} \cong k\left[b_{10}, b_{11}, b_{20}\right] \otimes \bigwedge\left(h_{10}, h_{11}, h_{20}\right) \Rightarrow H^{*}\left(P(1)^{*}\right) .
$$

For ease of notations, we write $b_{1 i}=b_{i}, h_{1 i}=h_{i}, b_{20}=u$ and $h_{20}=z$, and moreover $S=k\left[b_{0}, b_{1}\right]$. Hence in the new notation $E_{2}^{*, *}=S[u] \otimes \bigwedge\left(h_{0}, h_{1}, z\right)$, and $z \in E_{2}^{0,1}, u \in E_{2}^{0,2}, S \otimes \bigwedge\left(h_{0}, h_{1}\right)=E_{2}^{*, 0}$.

The first nonzero differential is $d_{2} z=h_{0} h_{1}$ and we get

$$
E_{3}^{*, *}=S[u]\left\{1, h_{0}, h_{1}, h_{0} z, h_{1} z, h_{0} h_{1} z\right\},
$$

where $S[u]\left\{1, h_{0}, \ldots\right\}$ means the free $S[u]$-module generated by $1, h_{0}, \ldots$ The next differential is

$$
d_{3} u=\widetilde{\mathcal{B}}\left(h_{0} h_{1}\right)=b_{0} h_{2}-b_{1} h_{1}=-b_{1} h_{1} .
$$

Since $d_{3}\left(u^{i}\right)=i u^{i-1} d_{3}(u)$, we get

$$
E_{4}^{* * *}=k\left[u^{p}\right] \otimes\left(A /\left(b_{1} h_{1}\right) \oplus H\left(A, b_{1} h_{1}\right)\left\{u, \ldots, u^{p-2}\right\} \oplus\left(\operatorname{Ker}\left(b_{1} h_{1}\right) \mid A\right)\left\{u^{p-1}\right\}\right)
$$

where $A=E_{3}^{*, 0} \oplus E_{3}^{*, 1}=S\left\{1, h_{0}, h_{1}, h_{0} z, h_{1} z, h_{0} h_{1} z\right\}$, and $H\left(A, h_{1} b_{1}\right)$ is the homology of $A$ defined by the differential $d x=h_{1} b_{1} x$ for each $x \in A$. In $S \otimes$ $\bigwedge\left(h_{0}, h_{1}\right) /\left(h_{0} h_{1}\right)$, we see $\operatorname{Ker}\left(b_{1} h_{1}\right)=S\left\{h_{0}, h_{1}\right\}$ and Image $\left(b_{1} h_{1}\right)=S\left\{h_{1} b_{1}\right\}$.

$$
E_{4}^{*, 2 i} \cong \begin{cases}S\left\{1, h_{0}\right\} \oplus S_{0}\left\{h_{1}\right\}, & i=0, \\ \left(S\left\{h_{0}\right\} \oplus S_{0}\left\{h_{1}\right\}\right) u^{i}, & 0<i \leq p-2, \\ S\left\{h_{0}, h_{1}\right\} u^{p-1}, & i=p-1,\end{cases}
$$

with $S_{0}=S /\left(b_{1}\right)$. The odd second degree parts are given for $a_{i}, a_{01} \in S$ by

$$
d_{3}\left(a_{0} h_{0}+a_{1} h_{1}+a_{01} h_{0} h_{1}\right) z u=a_{0} h_{0} h_{1} b_{1} z .
$$


Hence in $S\left\{h_{0} z, h_{1} z, h_{0} h_{1} z\right\}$, we get $\operatorname{Ker}\left(b_{1} h_{1}\right)=S\left\{h_{1} z, h_{0} h_{1} z\right\}$ and Image $\left(b_{1} h_{1}\right)=$ $S\left\{h_{0} h_{1} b_{1} z\right\}$. Thus we get

$$
E_{4}^{*, 2 i+1} \cong \begin{cases}\left(S\left\{h_{0}, h_{1}\right\} \oplus S_{0}\left\{h_{0} h_{1}\right\}\right) z, & i=0, \\ \left(S\left\{h_{1}\right\} \oplus S_{0}\left\{h_{0} h_{1}\right\}\right) u^{i} z, & 0<i<p-1, \\ S\left\{h_{1}, h_{0} h_{1}\right\} z u^{p-1}, & i=p-1 .\end{cases}
$$

Recall that the weight introduced in $\S 1$ is always a multiple of $p-1$. So divide it by $p-1$, and let us write it $\operatorname{wt}(x)$ so that $\operatorname{wt}\left(h_{i}\right)=\operatorname{wt}\left(b_{i-1}\right)=p^{i}$ and $\operatorname{wt}(z)=$ $p+1, \operatorname{wt}(u)=p(p+1)$. By Kudo's transgression theorem

$$
d_{2 p-1}\left(h_{1} b_{1} \otimes u^{p-1}\right)=\widetilde{\mathcal{B}} \widetilde{\mathcal{P}}^{\prime}\left(h_{1} b_{1}\right)=\widetilde{\mathcal{B}}\left(h_{1}\right) \mathcal{P}^{1}\left(b_{1}\right)=b_{1} b_{1}^{p}=b_{1}^{p+1} .
$$

Therefore $E_{2 p}^{*, *}$ is $b_{1}^{p+1}$-torsion. Hence there are $r \leq 2 p-1$ such that $d_{r}(x)=$ $b_{1}^{p+1} h_{1} z$. Since $\operatorname{wt}\left(b_{1}^{p+1} h_{1} z\right)=1 \bmod p$, we have $\operatorname{wt}(x)=1 \bmod p$. Hence $x=a h_{0} u^{i}$ or $x=a h_{1} u^{i} z$ for $a \in S$. However $x$ must be odd degree, since $d x$ is even degree. Thus $x=a h_{0} u^{i}$.

Since $|a|=2(p+1-i)$, let us write $a=\sum \lambda_{j} b_{0}^{j} b_{1}^{p+1-i-j}$. Then the weights are

$$
\begin{aligned}
\operatorname{wt}\left(b_{0}^{j} b_{1}^{p+1-i-j} h_{0} u^{i}\right) & =j p+p^{2}(p+1-j-i)+1+\left(p^{2}+p\right) i \\
& =(1-j) p^{2}+(j+i) p+1 \bmod p^{3} .
\end{aligned}
$$

This must be $\operatorname{wt}\left(b_{1}^{p+1} h_{1} z\right)=p^{2}+2 p+1$, hence $j=0 \bmod p$ and $i=2$. This is the only case. Hence we get $d_{4}\left(b_{1}^{p-1} h_{0} u^{2}\right)=b_{1}^{p+1} h_{1} z$, so we also see $d_{4}\left(h_{0} u^{2}\right)=b_{1}^{2} h_{1} z$. By similar arguments, we can prove

$$
d_{4}\left(h_{0} u^{i}\right)=b_{1}^{2} h_{1} z u^{i-2}, \quad 2 \leq i \leq p-1 .
$$

Indeed there is $x$ such that $d_{r}(x)=b_{1}^{p+1} h_{1} u^{j}, r \leq 2 p-2 j-1$, and we use $|u|=2$ and $\operatorname{wt}(u)=p^{2}+p$.

Remark 4.5. The formula (4.5) is also showed by the cohomology of Hopf algebra version of Theorem 3 in [L 2]. I. Leary proved the following; Let $0 \rightarrow Z / p \rightarrow G^{\prime} \rightarrow$ $G \rightarrow 1$ be a central extension of a finite group. We consider the induced HochschildSerre spectral sequence. Let us write $H^{*}(Z / p) \cong k[u] \otimes \bigwedge(z)$ and $d_{2} z=s, d_{3} u=s^{\prime}$. If $H^{*}(G)$ satisfies $s^{\prime} x=s x^{\prime}$ for some $x^{\prime} \in H^{*}(G)$, then $d_{4}\left(u^{n} x\right)=n(n-1) u^{n-2} z s^{\prime} x^{\prime}$. The formula (4.5) is the case with $x=h_{0}, x^{\prime}=b_{1}, s^{\prime}=b_{1} h_{1}, s=h_{0} h_{1}$.

Let $d^{\prime}(x)=d_{4}(x)$ for $x=h_{0} u^{i}, 2 \leq i \leq p-1$, and $d^{\prime}(x)=0$ for other $S$-module generators in $(4.3)$ and $(4.3)^{\prime}$. Let $E E_{5}^{*, *}=H\left(E_{4}^{*, *}, d^{\prime}\right)$. Of course $E_{5}^{*, *}$ is its subquotient. We get

$$
\begin{aligned}
E E_{5}^{*, j} \cong & \begin{cases}S\left\{1, h_{0}\right\} \oplus S_{0}\left\{h_{1}\right\}, & j=0, \\
\left(S\left\{h_{0}\right\} \oplus S_{0}\left\{h_{1}\right\}\right) u, & j=2, \\
S_{0}\left\{h_{1}\right\} u^{i}, & j=2 i, 2 \leq i \leq p-2, \\
S\left\{h_{1}\right\} u^{p-1}, & j=2 p-2,\end{cases} \\
& \begin{cases}\left(S\left\{h_{0}\right\} \oplus S /\left(b_{1}^{2}\right)\left\{h_{1}\right\} \oplus S_{0}\left\{h_{0} h_{1}\right\}\right) z, & j=1, \\
\left(S /\left(b_{1}^{2}\right)\left\{h_{1}\right\} \oplus S_{0}\left\{h_{0} h_{1}\right\}\right) z u^{i}, & j=2 i+1,1 \leq i<p-2, \\
\left(S\left\{h_{1}\right\} \oplus S_{0}\left\{h_{0} h_{1}\right\}\right) z u^{p-2}, & j=2 p-3, \\
S\left\{h_{1}, h_{0} h_{1}\right\} z u^{p-1}, & j=2 p-1 .\end{cases}
\end{aligned}
$$


The above computations are $E E_{5}^{*, 0} \cong E_{4}^{*, 0}, E E_{5}^{*, 2} \cong E_{4}^{*, 2}$ and $E E_{5}^{*, 4} \cong \operatorname{Ker} d^{\prime}$, $E_{5}^{*, 1} \cong E_{3}^{*, 1} / \operatorname{Im}\left(d^{\prime}\right)$. The differential is

$$
d^{\prime}: E_{4}^{*, 4}=\left(S\left\{h_{0} u\right\} \oplus S_{0}\left\{h_{1} u\right\}\right) \rightarrow E_{4}^{*, 1}=\left(S\left\{h_{0}, h_{1}\right\} \oplus S_{0}\left\{h_{0} h_{1}\right\}\right) z
$$

by $h_{0} u^{2} \mapsto b_{1}^{2} h_{1} z$; hence we know $E E_{5}^{*, 4}$ and $E E_{5}^{*, 1}$.

The elements $1, h_{0}, h_{0} u, h_{0} z$ are $S$-free. If $d_{r}(x)$ is $S$-free, then $x$ is also $S$-free. Other $S$-free modules are generated by

$$
B=k\left\{h_{1} u^{p-1}, h_{1} z u^{p-2}, h_{1} z u^{p-1}, h_{0} h_{1} z u^{p-1}\right\},
$$

so the differential which hit targets in $S\left\{1, h_{0}, h_{0} u, h_{1} z\right\}$ are $d_{r}$ with $r \leq 2 p-2$.

Lemma 4.7. All non $S$-free nonzero elements in $E E_{5}^{*, *}$ are also nonzero cycles in $E_{2 p-1}$.

Proof. From (4.6) we know all non $S$-free element in $E E_{5}^{*, *}$ are $b_{1}^{2}$-torsion. Suppose that the image $d_{r}$ contains a $b_{1}^{2}$-torsion element for $2 p-2 \geq r \geq 4$. From (4.6), $S$-module generators are written as $u^{d} z^{e} h_{0}^{f} h_{1}^{g}$. So we can write

$$
d_{r}\left(u^{d} z^{e} h_{0}^{f} h_{1}^{g}\right)=\sum \lambda b_{0}^{i} b_{1}^{j} u^{d^{\prime \prime}} z^{e^{\prime \prime}} h_{0}^{f^{\prime \prime}} h_{1}^{g^{\prime \prime}} \quad \text { with } j=0 \text { or } 1, \lambda \in Z / p .
$$

Then

$\operatorname{wt}($ the left of the above formula $)-\operatorname{wt}\left(u^{d^{\prime \prime}}\right)>\operatorname{wt}\left(u^{d-d^{\prime \prime}}\right)=\left(p^{2}+p\right)\left(d-d^{\prime \prime}\right)$, $\operatorname{wt}($ the right of the above formula $)-\operatorname{wt}\left(u^{d^{\prime \prime}}\right)=p i+p^{2} j+(p+1) e^{\prime \prime}+f^{\prime \prime}+p g^{\prime \prime}$.

By dimensional reason, we note $d-d^{\prime \prime} \geq i-1$ since other indexes $j, e^{\prime \prime}, \ldots$ are still 0 or 1 . If $d-d^{\prime \prime} \geq 2$, then the above two weights are not equal. When $d-d^{\prime \prime}=1$, $i=2$ and $j=1$, also by dimensional reason, we know $e=1, e^{\prime \prime}=f^{\prime \prime}=g^{\prime \prime}=0$. This case is also seen that the above two weights are not equal. Hence Image $\left(d_{r}\right)$ does not contain $b_{1}^{2}$-torsion elements.

Of course, the images of $b_{1}^{2}$-torsion elements are also $b_{1}^{2}$-torsion. Hence we get the lemma. q.e.d.

If $p \geq 5$, then of course $2 p-2>5$ and we have

Corollary 4.8. If $p \geq 5$, then $E E_{5}^{*, *} \cong E_{5}^{*, *} \cong E_{2 p-2}^{*, *}$.

For $p=3, E_{5}^{*, *}$ is a proper subquotient of $E E_{5}^{*, *}$ as stated below (4.9).

Since $E_{2 p}^{*, *}$ is $b_{1}^{p+1}$-torsion, for each $S$-free generator $a$ in $B$ which is not in the image $\left(d_{r}\right), r \leq 2 p-1$ by dimensional reason, we know ker $d_{r} \mid(S a)=0$ for some $r \leq 2 p-1$. The weight of free $S$-module generators are

$$
\operatorname{wt}\left(b_{1}^{p} h_{0}\right)=+1, \quad \operatorname{wt}\left(b_{1}^{p} h_{0} u\right)=p^{2}+p+1, \quad \operatorname{wt}\left(b_{1}^{p} h_{0} z\right)=p+2 \quad \bmod p^{3} .
$$

On the other hand the weight in $B$ are

$$
\begin{gathered}
\operatorname{wt}\left(h_{1} z u^{p-2}\right)=-p^{2}+1, \quad \operatorname{wt}\left(h_{1} z u^{p-1}\right)=1+p, \\
\operatorname{wt}\left(h_{0} h_{1} z u^{p-1}\right)=2+p \quad \bmod p^{3} .
\end{gathered}
$$

Therefore, we get the differential

$$
\begin{gathered}
d_{2 p-1}\left(h_{1} u^{p-1}\right)=b_{1}^{p} \text { Kudo's transgression, } \\
d_{2 p-1}\left(h_{0} h_{1} u^{p-1} z\right)=h_{0} b_{1}^{p} z \\
d_{2 p-2}\left(h_{1} u^{p-2} z\right)=b_{1}^{p-1} h_{0} \\
d_{2 p-2}\left(h_{1} u^{p-1} z\right)=b_{1}^{p-1} h_{0} u
\end{gathered}
$$


When $p=3, E_{5}=H\left(E E_{5}, d_{2 p-2}\right)$ here the differential $d_{2 p-2}$ is given in the above formulas. Recall $E E_{5} \cong E_{2 p-2}$ for $p \geq 5$. So for all $p \geq 3$, we have $E_{2 p-1} \cong H\left(E E_{5}, d_{2 p-2}\right)$. Thus we have

$$
\begin{gathered}
E_{2 p}^{*, j} \cong \begin{cases}S /\left(b_{1}^{p}\right)\{1\} \oplus S /\left(b_{1}^{p-1}\right)\left\{h_{0}\right\} \oplus S_{0}\left\{h_{1}\right\}, & j=0, \\
\left(S /\left(b_{1}^{p-1}\right)\left\{h_{0}\right\} \oplus S_{0}\left\{h_{1}\right\}\right) u, & j=2, \\
S_{0}\left\{h_{1}\right\} u^{i}, & j=2 i, 2 \leq i \leq p-2, \\
0, & j=p-1,\end{cases} \\
\begin{cases}\left(S /\left(b_{1}^{p-1}\right)\left\{h_{0}\right\} \oplus S /\left(b_{1}^{2}\right)\left\{h_{1}\right\} \oplus S_{0}\left\{h_{0} h_{1}\right\}\right) z, & j=1, \\
\left(S /\left(b_{1}^{2}\right)\left\{h_{1}\right\} \oplus S_{0}\left\{h_{0} h_{1}\right\}\right) z u^{i}, & j=2 i+1,1 \leq i \leq p-3, \\
S_{0}\left\{h_{0} h_{1}\right\} z u^{p-2}, & j=2 p-3, \\
0, & j=2 p-1 .\end{cases}
\end{gathered}
$$

Since $d_{2 p+1} u^{p}=d_{2 p+1} \widetilde{\mathcal{P}}^{1} u=\widetilde{P}^{1}\left(h_{1} b_{1}\right)=b_{1}^{p} h_{2}=0$, we have the following theorem.

Theorem 4.11. For all $p \geq 3$, we have isomorphisms

$$
E_{\infty}^{*, *} \cong E_{2 p}^{*, *} \cong(4.10) \text { and } E_{\infty}^{*, n}=E_{\infty}^{*, n+2 p} \text { for } n \geq 0 .
$$

Corollary 4.12. $\operatorname{gr} H^{*}\left(G(3)_{2}\right) \cong E_{\infty}^{* * *} \otimes k\left[b_{21}\right] \otimes \wedge\left(h_{21}\right)$.

Corollary 4.13. The cohomology $H^{*}(P(1))$ is multiplicatively generated by

$$
\begin{gathered}
h_{i}, b_{i}, g_{i}^{\prime}=\left[h_{i} z\right], k_{i}=\left[h_{i} u\right] \quad \text { for } i=0 \text { or } 1, \\
e_{j}=\left[h_{1} z u^{j-2}\right], f_{j}=\left[h_{1} u^{j-1}\right] \quad \text { for } 3 \leq j \leq p-1, \\
d_{p}^{\prime}=\left[h_{0} h_{1} z u^{p-2}\right] \quad \text { and } \quad v=\left[u^{p}\right] .
\end{gathered}
$$

Unfortunately, we do not decide the multiplicative structure completely. Here we give some partial results.

Lemma 4.14. Let $p \geq 5$. As $S=k\left[b_{0}, b_{1}\right]$-modules, $H^{*}(P(1)) \cong E_{\infty}^{* * *}$ in (4.10).

Proof. The proof is given by routine arguments using weight and degree. We give here the proofs for cases $b_{1} f_{j}=b_{1} d_{p}^{\prime}=0$. The proof of other cases are given similarly. First note

$$
\begin{gathered}
\operatorname{wt}\left(h_{0}\right)=\operatorname{wt}\left(k_{0}\right)=1, \quad \operatorname{wt}\left(h_{1}\right)=\operatorname{wt}\left(k_{1}\right)=0 \quad \bmod (p), \\
\operatorname{wt}\left(b_{i}\right)=0, \quad \operatorname{wt}\left(g_{0}^{\prime}\right)=2, \quad \operatorname{wt}\left(g_{1}^{\prime}\right)=1 \quad \bmod (p)
\end{gathered}
$$

for generators in $E_{\infty}^{*, j}, j \leq 2$. By (4.10), we get

$$
b_{1} f_{j}=\sum \alpha_{i} h_{i}+\beta_{i} k_{i} \quad \text { for } \alpha_{i}, \beta_{i} \in S .
$$

Since $\operatorname{wt}\left(b_{1} f_{j}\right)=0 \bmod p$, we know $\alpha_{0}=\beta_{0}=0$. For dimensional reason, $\left|\alpha_{1}\right|=$ $\left|\beta_{1}\right|+2=2 j$. The fact $\operatorname{wt}(u)>\operatorname{wt}\left(b_{1}\right)$ implies $\operatorname{wt}\left(b_{1} f_{j}\right)>\left|\alpha_{1} h_{1}\right|$ and $>\left|\beta_{1} k_{1}\right|$. Thus $b_{1} f_{j}=0$.

Next consider the case $b_{1} d_{p}^{\prime}$. Its weight is $2 \bmod p$. But there is no odd degree element of same weight in $E_{\infty}^{*, j}, j \leq 2$. Hence $b_{1} d_{p}^{\prime}=0$. q.e.d. 
Proposition 4.15. Let $p \geq 5$. The products $x \cdot y$ for generators $x, y \in\left\{h_{i}, g_{i}^{\prime}, k_{i}\right.$, $\left.e_{j}, f_{j}, d_{p}^{\prime}\right\}$ are zeros except for the following cases

$$
\begin{gathered}
h_{1} g_{0}^{\prime}=-h_{0} g_{1}^{\prime} \neq 0, \quad h_{0} k_{0}, h_{1} k_{0}, h_{0} k_{1}, h_{0} e_{j} \neq 0, \\
\left\{h_{1}, g_{1}^{\prime}, k_{1}\right\} \cdot\left\{e_{p-2}, e_{p-1}, f_{p-2}, f_{p-1}\right\}, e_{j} e_{p+1-j}, f_{j} f_{p-j}, f_{j} e_{p+1-j} .
\end{gathered}
$$

Remark. The author does not know whether the above elements are zero or nonzero. When $p=3$ the multiplications are more complicated, for example, we know $h_{0} g_{0}^{\prime} \neq$ $0, h_{0} g_{1}^{\prime} \neq 0, g_{0}^{\prime} g_{1}^{\prime} \neq 0$.

\section{Cohomology of $S(2)_{(2)}$}

In this section we compute the cohomology of $S(2)_{(2)} \cong k\left(G(3)\left(F_{p^{2}}\right)\right)^{*}$. Let $U$ be the maximal unipotent subgroup of $\mathrm{GL}_{3}$ generated by lower triangular matrices with diagonal elements 1 . Denote subgroups

$$
U_{\alpha}=\left(\begin{array}{ccc}
1 & & \\
* & 1 & \\
0 & 0 & 1
\end{array}\right), \quad U_{\beta}=\left(\begin{array}{lll}
1 & & \\
0 & 1 & \\
0 & * & 1
\end{array}\right), \quad U_{\gamma}=\left(\begin{array}{ccc}
1 & & \\
0 & 1 & \\
* & 0 & 1
\end{array}\right)
$$

which are isomorphic to $G_{a}$. The cohomology of $H^{*}(U)$ is studied in [K-S-T-Y 1] and $[\mathrm{Y}]$. We recall it before studying $H^{*}\left(G(3)\left(F_{p^{2}}\right)\right)$.

The spectral sequence induced from

$$
1 \rightarrow U_{\gamma} \rightarrow U \rightarrow U_{\alpha} \oplus U_{\beta} \rightarrow 1
$$

has the $E_{2}$-term

$$
E_{2}^{*, *} \cong H^{*}\left(U_{\alpha} \oplus U_{\beta}\right) \otimes H^{*}\left(U_{\gamma}\right) \Rightarrow H^{*}(U) .
$$

In [K-S-T-Y 1] $H^{*}\left(U_{2}\right)$ is computed completely. Moreover we know the spectral sequence (5.1) for low degree differentials. In the notation of [K-S-T-Y 1]

$$
\begin{gathered}
H^{*}\left(U_{\alpha} \oplus U_{\beta}\right)=\bigotimes_{i=0}^{\infty} k\left[y_{\alpha}(i+1), y_{\beta}(i+1)\right] \otimes \bigwedge\left(x_{\alpha}(i), x_{\beta}(i)\right), \quad y(i+1)=\widetilde{\mathcal{B}} x(i), \\
H^{*}\left(U_{\gamma}\right)=\bigotimes_{i=0}^{\infty} k\left[y_{\gamma}(i+1)\right] \otimes \bigwedge\left(x_{\gamma}(i)\right) .
\end{gathered}
$$

The first differential is $d_{2} x_{\gamma}(i)=x_{\alpha}(i) x_{\beta}(i)$. Hence

$$
E_{3}^{*, *} \cong \bigotimes_{i=0}^{\infty} P(i+1) \otimes H(i)
$$

with $P(i)=k\left[y_{\alpha}(i), y_{\beta}(i), y_{\gamma}(i)\right]$ and

$$
H(i)=k\left\{1, x_{\alpha}(i), x_{\beta}(i), x_{\alpha}(i) x_{\gamma}(i), x_{\beta}(i) x_{\gamma}(i), x_{\alpha}(i) x_{\beta}(i) x_{\gamma}(i)\right\} .
$$

The next differential is $d_{3} y_{\gamma}(i)=y_{\alpha}(i) x_{\beta}(i)-y_{\beta}(i) \cdot x_{\alpha}(i)$. The differential $d_{4}$ acts as $d_{4}\left(x_{\xi}(i) y_{\gamma}(i)^{j+2}\right)=\left(y_{\alpha}(i) x_{\beta}(i)-y_{\beta}(i) x_{\alpha}(i)\right) y_{\xi}(i) x_{\gamma}(i) y_{\gamma}(i)^{j}$ for $\xi=\alpha, \beta$. Moreover if $p>3$, then $d_{r}=0$ for $5 \leq r<2 p-2$. Thus these differentials are closed in $P(i) \otimes H(i)$. Hence if $p \geq 5$,

$$
E_{2 p-2}^{*, *} \cong\left(\bigotimes_{i=1} E(i)_{5}^{*, *}\right) \otimes H(0)
$$

where $E(i)_{5}^{*, *}$ is a subquotient of $P(i) \otimes H(i)$ which is given (5.6)(3) on page 89 in [K-S-T-Y 1]. For $p=3$, by arguments similar to (4.9)-(4.10), we see that $E_{2 p-1}$ is 
a subquotient of the right-hand side algebra of (5.3). We know all the differentials $d_{2 p-2}, d_{2 p-1}$. They are not closed in $P(i) \otimes H(i),(5.8),(5.9),(5.10)$ in [K-S-T-Y 1].

In particular we know for all primes $p \geq 3$

Proposition 5.4 (5.11 in [K-S-T-Y 1]). The $\bigotimes_{i=1}^{\infty} k\left[y_{\xi}(i)\right]$-module generators of $H^{*}(G(3))$ and in $\bigoplus_{s=0}^{2 p-1} E_{\infty}^{*, s}$ are given by elements in $H(0)$ and for $i \geq 1$

$$
\begin{gathered}
x_{\xi}(i), g_{\xi}(i)=\left[x_{\xi}(i) x_{\gamma}(i)\right], \quad k_{\xi}(i)=\left[x_{\xi}(i) y_{\gamma}(i)\right] \quad \text { for } \xi=\alpha \text { or } \beta, \\
d_{j}(i)=\left[x_{\alpha}(i) x_{\beta}(i) x_{\gamma}(i) y_{\gamma}(i)^{j-2}\right], \quad 3 \leq j \leq p, \\
c_{j}(i)=\left[\left(y_{\alpha}(i) x_{\beta}(i)-y_{\beta}(i) x_{\alpha}(i)\right) x_{\gamma}(i) y_{\gamma}(i)^{j-2}\right], \quad 3 \leq j \leq p-1 .
\end{gathered}
$$

Now consider the spectral sequence induced from (2.1)

$$
\begin{gathered}
E_{2}^{*, *} \cong k\left[b_{0}, b_{1}\right] \otimes \bigwedge\left(h_{0}, h_{1}\right) \otimes k\left[u_{0}, u_{1}\right] \otimes \bigwedge\left(z_{0}, z_{1}\right) \\
\Rightarrow H^{*}\left(G(3)\left(F_{p^{2}}\right)\right) \cong H^{*}\left(S(2)_{(2)}\right) .
\end{gathered}
$$

The map $i^{*}: H^{*}(U) \rightarrow H^{*}\left(U\left(F_{p^{2}}\right)\right) \rightarrow H^{*}\left(G(3)\left(F_{p^{2}}\right)\right)$ is given by

$$
\begin{aligned}
& i^{*} y_{\alpha}(\text { even })=i^{*} y_{\beta}(\text { odd })=b_{1}, \quad i^{*} y_{\beta}(\text { even })=i^{*} y_{\alpha}(\text { odd })=b_{0}, \\
& i^{*} x_{\alpha}(\text { even })=i^{*} x_{\beta}(\text { odd })=h_{0}, \quad i^{*} x_{\beta}(\text { even })=i^{*} x_{\alpha}(\text { odd })=h_{1}, \\
& i^{*} y_{\gamma}(\text { even })=u_{1}, i^{*} y_{\gamma}(\text { odd })=u_{0}, \quad i^{*} x_{\gamma}(\text { even })=z_{0}, i^{*} x_{\gamma}(\text { odd })=z_{1} .
\end{aligned}
$$

The first nonzero differential is

$$
d_{2} z_{0}=h_{0} h_{1}, d_{2} z_{1}=-h_{0} h_{1} .
$$

Hence $E_{3}^{*, *} \cong \widetilde{E}_{3}^{*, *} \otimes k\left[u_{0}+u_{1}\right] \otimes \bigwedge\left(z_{0}+z_{1}\right)$ with

$$
\widetilde{E}_{3}^{*, j} \cong \begin{cases}S\left\{1, h_{0}, h_{1}\right\} u^{i}, & j=2 i, \\ S\left\{h_{0}, h_{1}, h_{0} h_{1}\right\} u^{i} z, & j=2 i+1,\end{cases}
$$

where $u=u_{0}, z=z_{1}, h_{i}=h_{1 i}, S=k\left[b_{0}, b_{1}\right]$ with $b_{i}=b_{1 i}$.

Define $\widetilde{E}_{r+1}^{*, *}=H\left(\widetilde{E}_{r}^{*, *}, d_{r}\right)$ using the facts that $d_{r}\left(\widetilde{E}_{r}^{*, *}\right) \subset \widetilde{E}_{r}^{*, *}$, which is shown below. Then note that $E_{r}^{*, *} \cong \widetilde{E}_{r}^{*, *} \otimes k\left[u_{0}+u_{1}\right] \otimes \bigwedge\left(z_{0}+z_{1}\right)$.

The next differential is $d_{3} u_{1}=b_{0} h_{0}-b_{1} h_{1}$. Hence (see p. 88 in [K-S-T-Y 1])

$$
\begin{aligned}
\widetilde{E}_{4}^{*, j} \cong & \begin{cases}S\{1\} \oplus S\left\{h_{0}, h_{1}\right\} /\left(b_{0} h_{0}-b_{1} h_{1}\right), & j=0, \\
\left(S\left\{h_{0}, h_{1}\right\} /\left(b_{0} h_{0}-b_{1} h_{1}\right)\right) u^{i}, & 0<j=2 i<2(p-1), \\
S\left\{h_{0}, h_{1}\right\} u^{p-1}, & \end{cases} \\
& \begin{cases}\left(S\left\{h_{0}, h_{1}\right\} \oplus k\left\{h_{0} h_{1}\right\}\right) z, & j=1, \\
\left(S\left(b_{0} h_{0}-b_{1} h_{1}\right) \oplus k\left\{h_{0} h_{1}\right\}\right) z u^{i}, & 1<j=2 i+1<2(p-1), \\
S\left\{\left(b_{0} h_{0}-b_{1} h_{1}\right), h_{0} h_{1}\right\} x u^{p-1} .\end{cases}
\end{aligned}
$$

By naturality and (5.5) in [K-S-T-Y 1], the next differential is

$$
d_{4} h_{j} u^{i+2}=\left(b_{0} h_{0}-b_{1} h_{1}\right) b_{j+1} z u^{i} .
$$


Hence we get (see (5.6)(3) in [K-S-T-Y 1]) for $p>3$

$$
\begin{aligned}
\widetilde{E}_{5}^{*, j}= & \begin{cases}S\left\{1, h_{0}, h_{1}\right\} /\left(b_{0} h_{0}-b_{1} h_{1}\right), & j=0, \\
\left(S\left\{h_{0}, h_{1}\right\} /\left(b_{0} h_{0}-b_{1} h_{1}\right)\right) u, & j=2, \\
0, & 2<j=2 i<2(p-1), \\
S\left(b_{0} h_{0}-b_{1} h_{1}\right) u^{p-1}, & j=2(p-1),\end{cases} \\
& \begin{cases}S\left\{h_{0}, h_{1}, h_{0} h_{1}\right\} /\left(\left(b_{0} h_{0}-b_{1} h_{1}\right) b_{0},\right. & j=1, \\
\left.\quad\left(b_{0} h_{0}-b_{1} h_{1}\right) b_{1}\right) z, & 1<j=2 i+1<2 p-3, \\
k\left\{b_{0} h_{0}-b_{1} h_{1}, h_{0} h_{1}\right\} z u^{i}, & j=2 p-3, \\
\left.S\left\{b_{0} h_{0}-b_{1} h_{1}\right\} \oplus k\left\{h_{0} h_{1}\right\}\right) z u^{p-2}, & j=2 p-1 . \\
S\left\{b_{0} h_{0}-b_{1} h_{1}, h_{0} h_{1}\right\} z u^{p-1}, & \end{cases}
\end{aligned}
$$

When $p=3, E_{4}^{*, *}=E_{2 p-2}^{*, *}$ is given by (5.6) but $E_{5}^{*, *}=E_{2 p-1}^{*, *}$ is isomorphic to the homology of the right-hand side of (5.7) by the differential $d_{2 p-2}$ defined by the following two generators.

By naturality and (5.10) in [K-S-T-Y 1], we have

$$
\begin{gathered}
d_{2 p-2}\left(\left(b_{0} h_{0}-b_{1} h_{1}\right) z u^{p-2}\right)=b_{0}^{p} h_{1}-b_{1}^{p} h_{0}, \\
\left.d_{2 p-2}\left(b_{0} h_{0}-b_{1} h_{1}\right) z u^{p-1}\right)=\left(b_{0}^{p} h_{1}-b_{1}^{p} h_{0}\right) u .
\end{gathered}
$$

Kudo's transgression theorem (5.8) in [K-S-T-Y 1] gives

$$
\begin{gathered}
d_{2 p-1}\left(b_{0} h_{0}-b_{1} h_{1}\right) u^{p-1}=b_{0}^{p+1}-b_{1}^{p+1}, \\
d_{2 p-1}\left(h_{0} h_{1} z u^{p-1}\right)=\left(b_{0}^{p} h_{1}-b_{1}^{p} h_{0}\right) z .
\end{gathered}
$$

Therefore we get for all prime $p \geq 3$

$$
\begin{aligned}
\widetilde{E}_{2 p}^{*, j}= & \begin{cases}S /\left(b_{0}^{p+1}-b_{1}^{p+1}\right) & \\
\oplus S\left\{h_{0}, h_{1}\right\} /\left(b_{0} h_{0}-b_{1} h_{1}, b_{0}^{p} h_{1}-b_{1}^{p} h_{0}\right), & j=0, \\
S\left\{h_{0}, h_{1}\right\} /\left(b_{0} h_{0}-b_{1} h_{1}, b_{0}^{p} h_{1}-b_{1}^{p} h_{0}\right) u, & j=2, \\
0, & 2<j=2 i \leq 2 p-2 ;\end{cases} \\
& \begin{cases}S\left\{h_{0}, h_{1}, h_{0} h_{1}\right\} / & j=1, \\
\left(\left(b_{0} h_{0}-b_{1} h_{1}\right) b_{0},\left(b_{0} h_{0}-b_{1} h_{1}\right) b_{1},\right. & 1<j=2 i+1<2 p-3, \\
\left.b_{0}^{p} h_{1}-b_{1}^{p} h_{0}\right) z, & j=2 p-3, \\
k\left\{b_{0} h_{0}-b_{1} h_{1}, h_{0} h_{1}\right\} z u^{i}, & j=2 p-1 . \\
k\left\{h_{0} h_{1}\right\} z u^{p-2}, & j=2 p \\
0, & c i n\end{cases}
\end{aligned}
$$

Since $d_{2 p+1} u^{p}=b_{0}^{p} h_{1}-b_{1}^{p} h_{0}=0, u^{p}$ is a permanent cycle, so we get

Theorem 5.9. $E_{\infty}^{*, *} \cong \widetilde{E}_{2 p}^{*, *} \otimes k\left[u_{0}+u_{1}\right] \otimes \bigwedge\left(z_{0}+z_{1}\right)$.

Corollary 5.10. $H\left(S(2)_{(2)}\right)$ is multiplicatively generated by

$$
\begin{gathered}
v=\left[u^{p}\right], u^{\prime}=\left[u_{0}+u_{1}\right], z^{\prime}=\left[z_{0}+z_{1}\right], \\
h_{i}, b_{i}, g_{i}=\left[h_{i} z\right], k_{i}=\left[h_{i} u\right] \quad \text { for } i=0 \text { or } 1, \\
d_{j}=\left[h_{0} h_{1} z u^{j-2}\right] \quad \text { for } 4 \leq j \leq p, \\
c_{j}=\left[\left(b_{0} h_{0}-b_{1} h_{1}\right) z u^{j-2}\right] \quad \text { for } 4 \leq j \leq p-1 .
\end{gathered}
$$


$\left(\right.$ Note $d_{3}=\left[h_{0} h_{1} z u\right]=-g_{0} k_{1}$ and $\left.c_{3}=\beta\left(d_{3}\right)=k_{0} k_{1}.\right)$

Next consider its multiplicative structure. The weight is given modulo $\left(p^{2}-1\right)$ so that

$$
\begin{gathered}
\operatorname{wt}\left(h_{0}\right)=\operatorname{wt}\left(b_{1}\right)=1, \quad \operatorname{wt}\left(b_{0}\right)=\operatorname{wt}\left(h_{1}\right)=p, \\
\operatorname{wt}\left(g_{0}\right)=\operatorname{wt}\left(k_{0}\right)=2+p, \quad \operatorname{wt}\left(g_{1}\right)=\operatorname{wt}\left(k_{1}\right)=2 p+1 .
\end{gathered}
$$

By the weight and degree consideration, we easily see that

$$
h_{i} g_{i}=0, \quad h_{0} g_{1}+h_{1} g_{0}=0, \quad b_{i}\left(b_{0} g_{0}-b_{1} g_{1}\right)=0 \quad \text { for } p \geq 5 .
$$

Since $G(3)\left(F_{p^{2}}\right)$ is a finite group, we can also define the usual Bockstein $\beta=\sigma^{-1} \widetilde{\beta}$ and reduced powers $\mathcal{P}^{k}$, which preserve weight and Frobenius maps.

$$
\begin{array}{r}
\mathcal{B} h_{0}=b_{1}, \quad \mathcal{B} g_{0}=-k_{0} \quad \text { and } \mathcal{P}^{1} b_{0}=b_{1}^{p} \quad\left([\mathrm{Y}], \text { Lemma } 3.8, \text { note } z=z_{1}, u=u_{0}\right) \\
\left(\widetilde{\mathcal{B}} h_{0}=b_{0}, \widetilde{\mathcal{B}} g_{0}=k_{1}-h_{1} u^{\prime} \text { and } \widetilde{\mathcal{P}}^{1} b_{0}=b_{0}^{p} \text { with } u^{\prime}=\left[u_{0}+u_{1}\right]\right) .
\end{array}
$$

Using these operations, we know some relations. For example

$$
0=\beta\left(h_{0} g_{0}\right)=b_{1} g_{0}+h_{0} k_{0} \quad \text { for } p \geq 5 .
$$

We can get more information from the Bockstein operation, however we give here another argument using $H^{*}\left(U_{3}\left(F_{p}\right)\right)$.

From Proposition 5.4, the generators except for $z^{\prime}, u^{\prime}$ and $v$ are in the image from $H^{*}\left(U_{3}\right)$. Consider the diagram

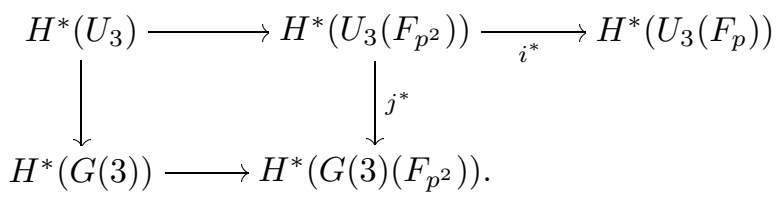

Ian Leary decided the multiplicative structure of $H^{*}\left(U_{3}\left(F_{p}\right)\right)$ completely (Theorem 4.14 and 4.15 in [L 1]), while it is quite complicated. In Leary's notation, $H^{*}\left(U_{3}\left(F_{p}\right)\right)$ is generated by elements $y, y^{\prime}, x, x^{\prime}, Y, Y^{\prime}, X, X^{\prime}, d_{4}, \ldots, d_{p}, c_{4}, \ldots$, $c_{p-1}, z$. The images of the restriction map $i^{*}$ are given

$$
\begin{gathered}
i^{*} x_{\alpha}(i)=y, i^{*} x_{\beta}(i)=y^{\prime}, \quad i^{*} y_{\alpha}(i)=x, \quad i^{*} y_{\beta}(i)=x^{\prime}, \\
i^{*} g_{\alpha}(i)=Y, \quad i^{*} g_{\beta}(i)=-Y^{\prime}, \quad i^{*} k_{\alpha}(i)=-X, \quad i^{*} k_{\beta}(i)=X^{\prime}, \\
i^{*} d_{j}(i)=d_{j}, \quad i^{*} c_{j}(i)=c_{j}, \quad \text { for } 4 \leq j \leq p-2, \\
i^{*} d_{p-1}(i)=d_{p-1}-x^{p-2} y-x^{p-2} y^{\prime}, \quad i^{*} d_{p}(i)=d_{p}-x^{p-2} X-x^{p-2} X^{\prime}, \\
i^{*} c_{p-1}(i)=c_{p-1}-x^{p-1}-x^{p-1} .
\end{gathered}
$$

Here $Y, Y^{\prime}, X$ are taken so that $y Y^{\prime}=y^{\prime} Y$ and $\beta(Y)=X$ but in our notation $x_{\alpha}(1) g_{\beta}(1)=-x_{\beta}(1) g_{\alpha}(1)$ and $\beta g_{\alpha}(1)=-k_{\beta}(1)$.

In $H^{*}\left(U_{3}\right)$, Kudo's transgression and related arguments show (see (5.11) in $[\mathrm{K}-\mathrm{S}-\mathrm{T}-\mathrm{Y} 1])$

$$
\begin{gathered}
y_{\alpha}(2) y_{\beta}(1)^{p}-y_{\beta}(2) y_{\alpha}(1)^{p}=0 \\
\left(y_{\alpha}(2) y_{\beta}(1)^{p-1}-y_{\beta}(2) y_{\alpha}(1)^{p-1}\right)\left\{x_{\xi}(1), g_{\xi}(1), k_{\xi}(1) \mid \text { for } \xi=\alpha \text { or } \beta\right\}=0 .
\end{gathered}
$$


Lemma 5.17. Let $x$ be a homogeneous (with respect to the weight) element in $H^{*}\left(U_{3}\right)$ represented in $\bigoplus_{j=0}^{2} E_{\infty}^{*, j}(1) \otimes k\left\{1, x_{\xi}(2), y_{\xi}(2)\right\}$ where $E_{\infty}^{* * *}(1)$ is the submodule in $E_{\infty}^{*, *}$ defined from elements in $P(1) \otimes H(1)$ in (5.2). If $x \in \operatorname{Ker} i^{*}$ and $\operatorname{wt}(x)<2 p^{2}(\alpha+\beta)$, then $x=0$.

Proof. First note that $\operatorname{Ker} i^{*} \mid\left(S(1)=k\left[y_{\alpha}(1), y_{\beta}(1)\right]\right)$ is the ideal $\left(y_{\alpha}(1)^{p} y_{\beta}(1)-\right.$ $\left.y_{\beta}(1) y_{\alpha}(1)^{p}\right)$. Each element in this ideal is nonhomogeneous with respect to the weight. Hence suppose that $x=a y_{\alpha}(2)+b$ with $0 \neq a \in S(1), b \in S(1)\left\{1, y_{\beta}(2)\right\}$. Since $x$ is homogeneous, we have

$$
x=c\left(a^{\prime} y_{\alpha}(2)+b^{\prime} y_{\beta}(1)^{p}\right) \quad \text { with } c \in S(1), a^{\prime} \in k\left[y_{\beta}(1)\right], b^{\prime} \in S(1)\left\{1, y_{\beta}(2)\right\} .
$$

For dimensional reasons $x=c^{\prime}\left(a^{\prime \prime} y_{\beta}(1)^{p-1} y_{\alpha}(2)+b^{\prime \prime} y_{\beta}(1)^{p}\right)$. Since $x$ is homogeneous and in $\operatorname{Ker} i^{*}$, we get

$$
x=c^{\prime \prime}\left(y_{\alpha}(1)^{p} y_{\beta}(2)-y_{\beta}(1)^{p} y_{\alpha}(2)\right) \quad \text { with } c^{\prime \prime} \in S(1) .
$$

Thus $x=0$ from (5.16). By similar arguments, we can also prove the lemma for the case $x \in S(1)\left\{x_{\xi}(1), g_{\xi}(1), k_{\xi}(1)\right\} \otimes\left\{1, x_{\xi}(2), y_{\xi}(2)\right\}$. q.e.d.

From this lemma, we can deduce all relations in $H^{*}\left(G(3)\left(F_{p}\right)\right)$. For example, there is a relation $y X=x Y$ for $p \geq 5$ in Theorem 2.14 [L 1]. Hence from Lemma 5.17 , we get $-x_{\alpha}(1) k_{\alpha}(1)=y_{\alpha}(1) g_{\alpha}(1)$. Hence $-h_{1} k_{1}=b_{0} g_{1}$, since

$$
i^{*} x_{\alpha}(1)=h_{1}, \quad i^{*} y_{\alpha}(1)=b_{0}, \quad i^{*} g_{\alpha}(1)=g_{1}, \quad i^{*} k_{\alpha}(1)=k_{1} .
$$

In Leary [L 1], we know

$$
c_{i} x= \begin{cases}0 & \text { for } i<p-1 \\ -x^{p} & \text { for } i=p-1\end{cases}
$$

So $i^{*}\left(c_{p-1}(1) y_{\alpha}(1)\right)=x^{\prime p-1} x$. Since $\operatorname{wt}\left(c_{p-1}(1)\right)=(p-1)(\alpha+\beta)$, we have

$$
c_{p-1}(1) y_{\alpha}(1)=y_{\beta}(1)^{p-1} y_{\alpha}(2) \text {. }
$$

Thus we have, in $H^{*}\left(G(3)\left(F_{p^{2}}\right)\right)$

$$
c_{i} b_{0}= \begin{cases}0 & \text { for } i<p-1 \\ b_{1}^{p} & \text { for } i=p-1\end{cases}
$$

Unfortunately we cannot directly deduce $H^{*}(P(1))$ from $H^{*}\left(S(2)_{(2)}\right)$ or $H^{*}(G(3))$. In the spectral sequence converging to $H^{*}(G(3))$, the differentials are given by

$$
\begin{gathered}
d_{2} z_{0}=h_{0} h_{1}, \quad d_{3} u_{0}=b_{0} h_{2}+b_{1} h_{1}, \\
d_{4} h_{1} u_{0}^{2}=\left(b_{0} h_{2}-b_{1} h_{1}\right) b_{0} z_{1} .
\end{gathered}
$$

Since $d_{3}\left(h_{0} u_{0}^{2}\right) \neq 0$ in this spectral sequence, $h_{0} u_{0}^{2}$ does not appear in $E_{4}$. However we already know in $(4.5), d_{3}\left(h_{0} u_{0}^{2}\right)=0$ and $d_{4}\left(h_{0} u_{0}^{2}\right)=b_{1}^{2} h_{1} z_{0}$ in the spectral sequence converging to $H^{*}(P(1))$.

We know some of $H^{*}\left(G(3)_{3}\right)$ from $H^{*}\left(G(3)\left(F_{p^{2}}\right)\right)$ and also a little about $H^{*}(P(1))$. Consider the map

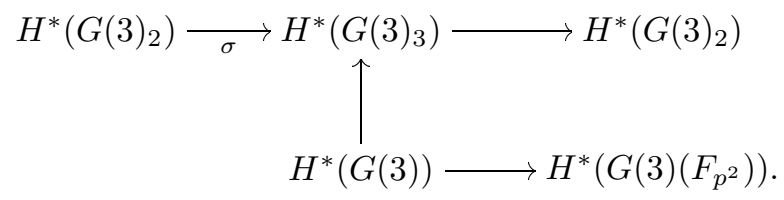


We give an incomplete set of generators of $H^{*}\left(G(3)_{3}\right)$ from those of $\sigma H^{*}\left(G(3)_{2}\right)$ and subsets of generators of $H^{*}\left(G(3)\left(F_{p^{2}}\right)\right)$ and $H^{*}\left(G(3)_{2}\right)$.

$$
\begin{gathered}
h_{i}, b_{i}, g_{i}^{\prime}=\left[h_{i} z_{0}\right], \quad \text { for } i=0 \text { or } 1, \\
g_{1}=\left[h_{1} z_{1}\right], \quad g_{2}=\left[h_{2} z_{1}\right], \quad k_{1}=\left[h_{1} u_{0}\right], \quad k_{2}=\left[h_{2} u_{0}\right], \\
d_{j}=\left[h_{1} h_{2} z u^{j-2}\right], \quad c_{j}=\left[b_{2} h_{2} z_{1} u_{0}^{j-2}\right], \\
h_{3}, b_{3}, g_{i}^{\prime} \cdot k_{i}, e_{j}, f_{j}, d_{p}^{\prime}, v, \text { and } z_{2}, u_{2}, z_{4}, u_{4} .
\end{gathered}
$$

Note that there are other generators, e.g., $g_{02}=\left[h_{0} z_{1}-h_{2} z_{0}\right]$.

Finally in this section, we give a relation in $H^{*}(P(1))$ from that of $H^{*}\left(U_{3}\left(F_{p}\right)\right)$. In [L 1] there is a relation $X y^{\prime}=2 x Y^{\prime}+x^{\prime} Y$ in $H^{*}\left(U_{3}\left(F_{p}\right)\right)$. So we get from Lemma 5.17

$$
k_{\alpha}(1) x_{\beta}(1)=-2 y_{\alpha}(1) g_{\beta}(1)+y_{\beta}(1) g_{\alpha}(1) \quad \text { in } H^{*}\left(U_{3}\right) .
$$

Hence $k_{1} h_{2}=-2 b_{0} g_{2}+b_{1} g_{1}$ in $H^{*}(G(3))$. Thus we have in $H^{*}\left(G(3)\left(F_{p^{2}}\right)\right)$

$$
k_{1} h_{0}=-2 b_{0} g_{0}+b_{1} g_{1} \text {. }
$$

Note that there is an element $k_{1} h_{0}$ from (5.20), which is represented in $E_{\infty}^{*, 1}$. Of course the restriction image of this element to $H^{*}\left(G(3)\left(F_{p^{2}}\right)\right)$ must be the righthand side of (5.21). Recall that

$$
\begin{gathered}
i^{*}: g_{02} \rightarrow\left[h_{0} z_{1}-h_{0} z_{0}\right]=2 g_{0}-h_{0}\left[z_{0}+z_{1}\right], \\
i^{*}: g_{1}^{\prime} \rightarrow\left[h_{1} z_{0}\right]=h_{1}\left[z_{0}+z_{1}\right]-g_{1} .
\end{gathered}
$$

Moreover $b_{0} h_{0}-b_{1} h_{1}=0$. Hence $i^{*}\left(-b_{0} g_{02}-b_{1} g_{1}\right)$ is the right-hand side of (5.21). Thus in $H^{*}(G(3)), h_{0} k_{1}=b_{0} g_{02}+b_{1} g_{1}^{\prime}$. Therefore we get in $H^{*}(P(1))$

$$
h_{0} k_{1}=b_{1} g_{1}^{\prime} .
$$

\section{Cohomology of the Morava stabilizer algebra}

In this section, we study the cohomology of the Morava stabilizer algebra $S(2)$, which is more easily computed than that of $S(2)_{(j)}$. Most results in this section about cohomology of $S(2)$ are already known by Ravenel [R 1]-[R 3] and by Henn $[\mathrm{H}]$ in more general forms.

Let us write the quotient algebra of $S(2)_{(j)}$ as $S(2)_{(k, j)}$, letting $t_{s}=0$ for $1 \leq$ $s \leq k-1$. The filtration induced from injections

$$
S(2)_{(2,2)} \rightarrow S(2)_{(2,3)} \rightarrow \cdots \rightarrow S(2)_{(2, j)} \rightarrow \cdots \rightarrow S(2)_{(2, \infty)}
$$

gives a spectral sequence similar to (2.10), such that

$$
E_{2}=\bigotimes_{i \geq 2, k \in Z / 2}^{\infty} k\left[b_{i k}\right] \otimes \bigwedge\left(h_{i k}\right) \Rightarrow H^{*}\left(S(2)_{(2, \infty)}\right) .
$$

From the property of the coproduct (1.5) and $t_{1}=0$, we know

$$
d_{2} h_{i+2,0}=b_{i, 1} \bmod \left(h_{j, k}, b_{j-1, k} \mid j \leq i, k \in Z / 2\right) \quad \text { for } i \geq 2 .
$$

Hence we have (see Theorem 6.3.7 in [R 1])

$$
E_{3} \cong \bigotimes_{k \in Z / 2} \bigwedge\left(h_{2 k}, h_{3 k}\right) \cong H^{*}\left(S(2)_{(2, \infty)}\right)
$$


Next consider the spectral sequence

$$
E_{2} \cong \bigotimes_{k \in Z / 2} k\left[b_{1 k}\right] \otimes \bigwedge\left(h_{1 k}, h_{2 k}, h_{3 k}\right) \Rightarrow H^{*}\left(S(2)_{(1, \infty)}=S(2)\right) .
$$

Since the algebra $B=\bigotimes_{k \in Z / 2} k\left[b_{1 k}\right] \otimes \bigwedge\left(h_{1 k}, h_{2 k}\right)$ is closed under the differential from the coproduct (1.5), we get the spectral sequences

$$
E_{2}^{\prime \prime} \cong H(B, d) \otimes \bigotimes_{k \in Z / 2} \bigwedge\left(h_{3 k}\right) \Rightarrow H^{*}(S(2))
$$

where the differential in $B$ is $d_{2} h_{20}=h_{10} h_{11}$. Hence

$$
A=H(B, d) \cong k\left[b_{0}, b_{1}\right]\left\{1, h_{0}, h_{1}, g_{0}, g_{1}, h_{0} g_{1}\right\} \otimes \bigwedge\left(z^{\prime}\right) \quad \text { with } z^{\prime}=\left(h_{20}+h_{21}\right) .
$$

Here $g_{k}=\left[h_{k} z\right]=\left[h_{1 k} h_{20}\right]$ as in Corollary 5.10. The quotient map $S(2) \rightarrow S(2)_{(2)}$ induces the map $H^{*}\left(S(2)_{(2)}\right) \rightarrow H^{*}(S(2))$. For an element $x$ in $(6.5), d_{r}(x)=0$ for all $r$ in the spectral sequence in (6.5), because $x$ is represented as an image from $H^{*}\left(S(2)_{(2)}\right)$ by Corollary 5.10 .

When $p \geq 5$, the relation in $A$ are $h_{i} g_{i}=g_{i} g_{i+1}=h_{0} g_{1}+h_{1} g_{0}=0$ (5.11) and the differentials in $E_{2}^{\prime \prime}$ are $d_{2} h_{30}=b_{1}, d_{2} h_{31}=b_{0}$. Thus we get

Theorem 6.7. (Ravenel) For $p \geq 5$,

$$
H^{*}(S(2)) \cong k\left\{1, h_{0}, h_{1}, g_{0}, g_{1}, h_{0} g_{1}\right\} \otimes \bigwedge\left(z^{\prime}\right) .
$$

Corollary 6.8. For $p \geq 5$, the inflation map $H^{*}\left(S(2)_{(2)}\right) \rightarrow H^{*}(S(2))$ is epic.

When $p=3$, the multiplicative structure of $A$ is very different

$$
h_{i} \tilde{g}_{i}=-b_{i} h_{i+1}, h_{0} \tilde{g}_{1}-h_{0} \tilde{g}_{1}=0, \tilde{g}_{i}^{2}=-b_{i} \tilde{g}_{i+1}, \tilde{g}_{0} \tilde{g}_{1}=b_{0} b_{1} .
$$

Here we take new generators $\tilde{g}_{0}=\left[h_{0}\left(z_{0}-z_{1}\right)\right]=g_{0}+h_{0} z^{\prime}, \tilde{g}_{1}=\left[h_{1}\left(z_{1}-z_{0}\right)\right]=$ $-g_{1}-h_{1} z^{\prime}$ so that $\sigma\left(\tilde{g}_{0}\right)=\tilde{g}_{1}$. This is proved by using the Massey product (page 294 in [R 2]) or by using Lemma 5.17 and the result of Leary (Theorem 2.15 in [L 1]) by the map $j^{*} i^{*-1}$ in (5.14), exchanging $y$ (resp. $\left.y^{\prime}, x, x^{\prime}, Y, Y^{\prime}\right)$ with $h_{1}$ (resp. $h_{0}, b_{0}, b_{1}, g_{1},-g_{0}$ ).

For $p=3$, the differentials in $E^{\prime \prime}$ are given by $d_{2} h_{3 i}=b_{i+1}-\tilde{g}_{i}$ ([R 2]). This is also proved by the definition of coproduct (see [R 2]). We can prove that $b_{1}-\tilde{g}_{0}$ is a nonzero divisor in $A$, e.g. for $x=a+a_{0} \tilde{g}_{0}+a_{1} \tilde{g}_{1} \in A, a, a_{i} \in k\left[b_{0}, b_{1}\right]$,

$$
\begin{aligned}
d\left(x h_{30}\right) & =\left(a+a_{0} \tilde{g}_{0}+a_{1} \tilde{g}_{1}\right)\left(\tilde{g}_{0}-b_{1}\right) \\
& =\left(a-a_{0} b_{1}\right) \tilde{g}_{0}+\left(-a_{0} b_{0}-a_{1} b_{1}\right) \tilde{g}_{1}+\left(a_{1} b_{0}-a\right) b_{1},
\end{aligned}
$$

using (6.9), hence if $d\left(x h_{30}\right)=0$, then $a=a_{0}=a_{1}=0$.

Hence for the spectral sequence $E^{\prime \prime}$, we only need to know the homology of $A /\left(b_{1}-\tilde{g}_{0}\right)$ with the differential $b_{0}-\tilde{g}_{1}=d_{2}\left(h_{31}\right)$.

$$
E_{3}^{\prime \prime} \cong A /\left(b_{1}-\tilde{g}_{0}, b_{0}-\tilde{g}_{1}\right) \oplus\left(\operatorname{Ker}\left(b_{0}-\tilde{g}_{1}\right) \mid A /\left(b_{1}-\tilde{g}_{0}\right)\right)\left\{h_{31}\right\} .
$$

The algebra $A /\left(b_{1}-\tilde{g}_{0}\right)$ is generated as a $k\left[b_{0}, b_{1}\right]$-module by $\left\{1, h_{0}, h_{1}, \tilde{g}_{1}, h_{0} \tilde{g}_{1}\right\}$ with the relation

$$
\begin{aligned}
h_{0} b_{1} & =-b_{0} h_{1}, \quad h_{1} \tilde{g}_{1}=-b_{1} h_{0}, \quad h_{1} b_{1}-h_{0} \tilde{g}_{1}=0, \\
b_{1}^{2} & =-b_{0} \tilde{g}_{1}, \quad \tilde{g}_{1}^{2} k=-b_{1}^{2}, \quad b_{1} \tilde{g}_{1}=b_{0} b_{1}
\end{aligned}
$$


from (6.9). Hence we have an isomorphism of $k\left[b_{0}\right]$-modules

$$
A /\left(b_{1}-\tilde{g}_{0}\right) \cong k\left[b_{0}\right]\left\{1, h_{0}, h_{1}, b_{1}, \tilde{g}_{1}, h_{0} \tilde{g}_{0}\right\} \otimes \bigwedge\left(z^{\prime}\right) .
$$

We easily see that $h_{1}, b_{1}, \tilde{g}_{1} \in \operatorname{Ker}\left(b_{0}-\tilde{g}_{1}\right)$ in $A /\left(b_{1}-\tilde{g}_{0}\right)$, e.g.,

$$
h_{1}\left(\tilde{g}_{1}-b_{0}\right)=-b_{1} h_{0}-h_{1} b_{0}=0, \quad \tilde{g}_{1}\left(\tilde{g}_{1}-b_{0}\right)=-b_{1}^{2}+b_{1}^{2}=0 .
$$

For each element $0 \neq x \in k\left[b_{0}\right]\left\{1, h_{0}\right\}$, it is easily proved $\left(\tilde{g}_{1}-b_{0}\right) x \neq 0$. Thus we get

Proposition 6.11. (Henn [H], [G-S-S]) When $p=3$, as $k\left[b_{0}\right]$-modules

$$
H^{*}(S(2)) \cong k\left[b_{0}\right]\left\{1, h_{0}, h_{1}, b_{1}, a, c_{0}^{\prime}, c_{1}^{\prime}, b_{1} a\right\} \otimes \bigwedge\left(z^{\prime}\right)
$$

with $a=\left[h_{1} h_{31}\right], c_{0}^{\prime}=\left[b_{1} h_{31}\right], c_{1}^{\prime}=\left[g_{1} h_{31}\right]$.

The fact that $a, c_{i}^{\prime}$ are permanent cycles in (6.5) is also proved from Corollary 6.14 below.

Let us write as $G S_{(j)}$ an algebraic group with the group ring $k\left(G S_{(j)}\left(F_{p^{2}}\right)\right) \cong$ $S(2)_{(j)}$. Here we construct this group only for $j=3$. Recall that the second Witt group $W_{2}$ is defined by $W_{2}(A)=A \times A$ for each commutative $k$-algebra $A$ with the sum

$$
\left(a_{1}, a_{2}\right)+\left(a_{1}^{\prime}, a_{2}^{\prime}\right)=\left(a_{1}+a_{1}^{\prime}, a_{2}+a_{2}^{\prime}-C_{p}\left(a_{1}, a_{1}^{\prime}\right)\right) \text { for } a_{i}, a_{i}^{\prime} \in A,
$$

(see (1.5) for the definition of $C_{p}$ ). From equality (1.5), the coproduct is $\psi\left(t_{3}\right)=$ $\sum_{j=0} t_{j} \otimes t_{3-j}^{p^{j}}-C_{p}\left(t_{1}^{p} \otimes 1+1 \otimes t_{1}^{p}\right)$. Hence the coordinate ring of $G S_{(3)}$ is given by

$$
\begin{aligned}
k[G(4)] \otimes k\left[W_{2}\right] & \rightarrow k\left[t_{1}, t_{2}, t_{3}, x_{1}, x_{2}\right] /\left(t_{1}=x_{1}\right) \\
& \supset k\left[t_{1}, t_{2}, t_{3}+x_{2}^{p}\right] \cong k\left[G S_{(3)}\right] .
\end{aligned}
$$

Let $G_{1}(A)$ (resp. $\left.G_{2}(A)\right)$ be the subgroup of $\left(G(4) \times W_{2}\right)(A)$ generated by

(6.12) $\left\{g\left(a_{1}, a_{2}, a_{3}\right) \times\left(a_{1}, b_{2}\right) \mid a_{i}, b_{2} \in A\right\} \quad\left(\right.$ resp. $\left.\left\{g\left(0,0,-b_{2}^{p}\right) \times\left(0, b_{2}\right) \mid b_{2} \in A\right\}\right)$.

Since $\operatorname{Hom}(k[G], A) \cong G(A)$, we know $G S_{(3)} \cong G_{1} / G_{2}$.

Therefore we have a short exact sequence of groups

$$
0 \rightarrow G_{a} \rightarrow G S_{(3)} \rightarrow G S_{(2)} \rightarrow 1 .
$$

The induced spectral sequence of $F_{p^{2}}$-points is

$$
E_{2}^{*, *}=H^{*}\left(S(2)_{(2)}\right) \otimes H^{*}\left(F_{p^{2}}\right) \Rightarrow H^{*}\left(S(2)_{(3)}\right) .
$$

For $p=3$, since $d_{2}\left(h_{3 i}\right)=b_{i+1}-\tilde{g}_{i}$, for $h_{3 i} \in H^{*}\left(F_{p^{2}}\right)$, the following elements are permanent cycles in the above spectral sequence

$$
a=\left[h_{1} h_{31}-h_{0} h_{30}\right], \quad c_{1}^{\prime}=\left[b_{1} h_{31}+\tilde{g}_{1} h_{30}\right] \quad \text { and } \quad c_{0}^{\prime}=\left[\tilde{g}_{1} h_{31}-\tilde{g}_{0} h_{30}\right] .
$$

Hence we have

Corollary 6.14. The inflation map $H^{*}\left(S(2)_{(3)}\right) \rightarrow H^{*}(S(2))$ is epic.

The relations (6.9) are given in $S(2)_{(2)}$ and $d_{2}\left(h_{3 i}\right)=b_{i+1}-\tilde{g}_{i}$ for the spectral sequence (6.13). Hence $b_{0}^{2}+b_{1}^{2}=0$ in $H^{*}\left(S(2)_{(3)}\right)$. Thus for the map $\pi: G S_{(3)}\left(F_{9}\right) \rightarrow G(3)\left(F_{9}\right)$, the variety is

$$
\left(\pi^{*} k\left[b_{1}, b_{0}\right]\right)(k) \cong \operatorname{Var}\left(b_{1}^{2}+b_{0}^{2}\right)=V_{+} \cup V_{-} \subset k\left\{b_{0}, b_{1}\right\}
$$

where $V_{*}=\left\{b_{0}+i b_{1}\right\}$ and $V_{-}=\left\{b_{0}-i b\right\}$. Hence there are subgroups $A_{+}, A_{-}$ which are isomorphic to $Z / 3$ but not conjugate to each other in $G S_{(3)}\left(F_{9}\right)$. 
To seek $A_{ \pm}$, we recall the definition of $b_{i}$. Identify $G(2)\left(F_{9}\right)=F_{9}$, let $x_{0}$ (resp. $\left.x_{1}\right)$ in $H^{1}\left(G(2)\left(F_{9}\right)\right)=\operatorname{Hom}\left(F_{9}, k\right)$ be the element defined by the natural nonzero map

$$
\left.F_{9}=Z / 3 \oplus Z / 3=\langle 1\rangle \oplus\langle i\rangle \rightarrow\langle 1\rangle \rightarrow k \quad \text { (resp. } F_{9} \rightarrow\langle i\rangle \rightarrow k\right) .
$$

Let $y_{i}=\beta\left(x_{i}\right)$. Recall that $h_{0}$ (resp. $\left.h_{1}\right)$ is the element induced from the identity (resp. the Frobenius map) in $\operatorname{Hom}\left(F_{9}, F_{9}\right)=H^{1}\left(F_{9}, F_{9}\right)$. Hence

$$
\begin{gathered}
h_{0}=x_{0}+i x_{1}, \quad h_{1}=x_{0}+i^{3} x_{1}=x_{0}-i x_{1} \quad(\text { see }(3.4) \text { in }[\mathrm{Y}]), \\
b_{0}=\tilde{\beta}\left(h_{0}\right)=y_{0}-i y_{1} \quad \text { and } \quad b_{1}=y_{0}+i y_{1} .
\end{gathered}
$$

Since $V\left(b_{0}+i b_{1}\right)=V\left(y_{0}-y_{1}\right)=\operatorname{Ker}\left(H^{*}\left(G(2)=F_{9}\right) \rightarrow H^{*}\left(A_{+}\right)\right)$, we know $A_{+}=\langle 1+i\rangle$ (resp. $\left.A_{-}=\langle 1-i\rangle\right)$ in $G(2)$. Therefore in $G S_{(3)}$

$$
A_{+}=\langle g(1+i, 0,0) \times(1+i, 0)\rangle, \quad A_{-}=\langle g(1-i, 0,0) \times(1-i, 0)\rangle,
$$

indeed, $3 \cdot g(\alpha, 0,0)=g(0,0,-\alpha)$ in $G(4)$ and $3 \cdot(\alpha, 0)=\left(0, \alpha^{3}\right)$ in $W_{2}$ for $\alpha=1 \pm i$, hence $A_{ \pm}$has order 3 .

For $x_{ \pm} \in H^{1}\left(A_{ \pm}\right)$and $y_{ \pm}=\beta\left(x_{ \pm}\right)$, we see $x_{0}\left|A_{ \pm}=x_{ \pm}, x_{1}\right| A_{ \pm}= \pm x_{ \pm}$and hence

$$
\begin{aligned}
h_{0} \mid A_{ \pm}= & \left(x_{0}+i x_{1}\right)\left|A_{ \pm}=(1 \pm i) x_{ \pm}, \quad h_{1}\right| A_{+}=(1 \mp i) \mid x_{ \pm}, \\
& b_{0}\left|A_{+}=(1 \mp i) y_{ \pm}, \quad b_{1}\right| A_{ \pm}=(1 \pm i) y_{ \pm} .
\end{aligned}
$$

Note that $b_{0} \pm i b_{1} \mid A_{ \pm}=0$.

Lemma 6.15. If $x \in k\left[b_{0}\right]\left\{b_{0}, b_{1}\right\} \subset H^{*}\left(S(2)_{(3)}\right)$ and $x\left|A_{+}=x\right| A_{-}=0$, then $x=0$.

Proof. Each element $x$ is written $x=\lambda b_{0}^{s}+\mu b_{1} b_{0}^{s-1}$. Then

$$
x\left|A_{+}=(\lambda+i \mu)(1+i)^{s} y_{+}^{s}, \quad x\right| A_{-}=(\lambda-i \mu)(1-i)^{s} y_{-}^{s} .
$$

Therefore, we see the lemma. q.e.d.

Theorem 6.16. (Henn $[\mathrm{H}]$, Golbanov-Siegel-Symonds $[\mathrm{G}-\mathrm{S}-\mathrm{S}])$ In $H^{*}(S(2))$, there are relations

$$
\begin{gathered}
a^{2}=a h_{0}=a h_{1}=a c_{0}^{\prime}=a c_{1}^{\prime}=0, \quad c_{0} h_{0}=c_{1} h_{1}=a b_{1}, \\
c_{0} h_{1}=c_{1} h_{0}=a b_{0}, \quad c_{0} c_{1}=0 .
\end{gathered}
$$

Proof. In the spectral sequence (6.5) or (6.13), we always know $a^{2}=0 \in E_{\infty}^{*, *}$. Hence $a^{2} \in k\left[b_{0}\right]\left\{1, b_{1}, a, a b_{1}\right\}$. Consider the subgroup

$$
Z / 3 \cong B_{ \pm}=\langle g(0,0,1 \pm i) \times(0,0)\rangle \subset F_{9} \subset G S_{(3)}\left(F_{9}\right) .
$$

Let $w_{ \pm} \in H^{1}\left(B_{ \pm}\right)$be the element induced from the identity of $\operatorname{Hom}\left(B_{ \pm}, B_{ \pm}=\right.$ $Z / 3)$. Then we see $h_{30} \mid B_{ \pm}=(1+i) w_{ \pm}$and $h_{31} \mid B_{ \pm}=(1 \mp i) w_{ \pm}$by arguments similar to the case $A_{ \pm}$. Since $a=\left[h_{1} h_{31}-h_{0} h_{30}\right]$, the restriction maps of $a$ to groups $A_{+} \oplus B_{+}$are

$$
\begin{aligned}
a \mid A_{+} \oplus B_{+} & =\left((1-i)^{2}-(1+i)^{2}\right) x_{+} w_{+} \\
& =-i x_{+} w_{+} \in k\left[y_{+}\right] \otimes \bigwedge\left(x_{+}, w_{+}\right) \subset H^{*}\left(A_{+} \oplus B_{+}\right) .
\end{aligned}
$$

Similarly $a \mid A_{-} \oplus B_{-}=i x_{-} w_{-}$. Both cases $a^{2}\left|A_{+} \oplus B_{+}=a^{2}\right| A_{-} \oplus B_{-}=0$. 
Let us write $a^{2}=s_{00} b_{0}^{2}+s_{10} b_{1} b_{0}+s_{0 a} b_{0} a+s_{1 a} b_{1} a$. Then

$$
\begin{aligned}
a^{2} \mid A_{ \pm} \oplus B_{ \pm}= & \left(s_{00}\left(1_{\mp} i\right)^{2}+s_{10}(1-i)(1+i)\right) y_{ \pm}^{2} \\
& \mp i\left(s_{0 a}(1 \mp i)+s_{1 a}(1 \pm i)\right) y_{ \pm} x_{ \pm} w_{ \pm} .
\end{aligned}
$$

Thus all $s_{j}, s_{j a}$ are zero, that is $a^{2}=0$ in $H^{*}\left(S(2)_{(3)}\right)$.

The other cases are proved by the same arguments, using the fact that $\tilde{g}_{i} \mid A_{ \pm}=$ $b_{i+1} \mid A_{ \pm}$since $\tilde{g}_{0} \tilde{g}_{1}=b_{0} b_{1}, h_{0} b_{0}=h_{1} b_{1}$ and $h_{0} \tilde{g}_{1}=h_{1} \tilde{g}_{0}$ in $H^{*}\left(S(2)_{(2)}\right)$ (6.9). Therefore we get relations in $H^{*}\left(S(2)_{(3)}\right)$, so in $H^{*}(S(2))$. q.e.d.

\section{REFERENCES}

[E] L. Evens, Cohomology of groups, Oxford Univ. Press, 1991. MR 93i:20059

[G-S-S] V. Gorbounov, S. Siegel, and P. Symonds, The cohomology of the Morava stabilizer group $S_{2}$ at the prime 3, Preprint 1994.

[K-S-T-Y 1] M. Kaneda, N. Shimada, M. Tezuka, and N. Yagita, Cohomology of infinitesimal algebraic groups, Math. Z. 205 (1990), 61-95. MR 91k:20048

[K-S-T-Y 2] _ Representations of the Steenrod algebra, J. Algebra 155 (1993), 435-453. MR 94b:55025

[H] H-W. Henn, On the $\bmod p$ cohomology of profinite groups of positive $p$ rank, Preprint, 1994.

[M-W] H. Miller and C. Wilkerson, Vanishing lines for modules over the Steenrod algebra, J. Pure Appl. Algebra 22 (1981), 293-307. MR 82m:55024

[L 1] I. Leary, The cohomology of certain finite groups, Thesis, Cambridge Univ., 1990.

[L 2] _ A differential in the Lyndon-Hochschild-Serre spectral sequence, J. Pure Appl. Algebra 88 (1993), 155-168. MR 94m:20102

[Li] A. Liulevicius, The factorization of cyclic reduced power by secondary cohomology operation, Mem. Amer. Math. Soc. 42, 1962. MR 31:6226

[P-Y] C. Peterson and N. Yagita, Rational cohomology of Witt groups, Math. Z. 224 (1997), 665-676. CMP 97:13

[Q] D. Quillen, The spectrum of an equivariant cohomology ring. I, II, Ann. of Math. 94 (1971), 549-572; 573-602. MR 45:7743

[R 1] D. Ravenel, The structure of Morava stabilizer algebras, Invent. Math. 37 (1976), 109-120. MR 54:8632

[R 2] The cohomology of the Morava stabilizer algebra, Math. Z. 152 (1977), 287-297. MR 55:4170

[R 3] Complex cobordism and stable homotopy groups of spheres, Academic Press, New York, 1986. MR 87j:55003

[S-I] N. Shimada and A. Iwai, On the cohomology of some Hopf algebras, Nagoya Math. J. 30 (1967), 103-111. MR 35:6731

[T] M. Tezuka, Cohomology of unipotent algebraic and finite groups and the Steenrod algebra, Math. Z. 216 (1994), 45-67. MR 95c:20064

[Y] N. Yagita, Frobenius operations and cohomology of $\mathrm{GL}_{3}\left(F_{q}\right)$, Comm. Algebra 16 (1988), 989-1016. MR 89c:20077

Faculty of Education, Ibaraki University, Mito, Ibaraki, Japan

E-mail address: yagita@mito.ipc.ibaraki.ac.jp 